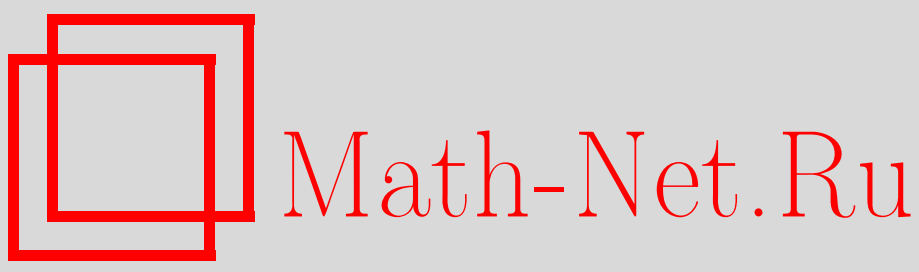

М. М. Гриненко, Бирациональные свойства пучков поверхностей дель Пеццо степеней 1 и 2. II, Матем. сб., 2003, том 194, номер 5, 31-60

DOI: https://doi.org/10.4213/sm734

Использование Общероссийского математического портала Math-Net.Ru подразумевает, что вы прочитали и согласны с пользовательским соглашением

http://www . mathnet.ru/rus/agreement

Параметры загрузки:

IP: 3.93 .64 .190

26 апреля 2023 г., 10:52:56 
УДК 512.6

М.М. Гриненко

\title{
Бирациональные свойства пучков поверхностей дель Пеццо степеней 1 и 2 . II
}

\author{
В работе доказывается бирациональная жесткость некоторых типов расслое- \\ ний на поверхности дель Пеццо степени 2. \\ Библиография: 6 названий.
}

Настоящая работа продолжает исследования, ранее начатые автором в статье [1], в которой было завершено решение проблемы бирациональной жесткости для неособых расслоений Мори на поверхности дель Пеццо степени 1 и почти завершено для степени 2. Оставшиеся 5 случаев степени 2 , перечисленные в леммах 3.3 и 3.4 указанной статьи, были предположены жесткими. Ниже доказывается, что на самом деле два из этих пяти случаев не реализуются, а оставшшеся три действительно являются жесткими. К сожалению, пока их жесткость удается показать только при некоторых условиях общности положения, но автор не сомневается, что эти условия могут быть опушены. Добавим, что для пучков кубических поверхностей проблема бирациональной жесткости значительно сложнее. Соответствующую информацию можно найти в работах [2], [3].

Технической основой статьи служит метод максимальных особенностей в виде, предложенном А. В. Пухликовым в [4]. Особенностью работы является то, что мы получаем сильные оценки снизу на "хвост" квадратичного неравенства (обычно отбрасьваемьй) через оценки сверху и снизу на кратности линейной системы. Последнее достигается систематическим применением одной и той же процедуры: ограничиваем линейную систему на слой, содержащий максимальную особенность, смотрим на кратности, затем делаем то же на первом “этаже” разрешения максимальной особенности для собственных прообразов, затем - на втором и т.д., после чего комбинируем оценки на кратности ограничений.

Настоящая статья организована следующим образом. Первьй параграф содержит два полезных факта, относящихся к методу максимальных особенностей в целом. Во втором напоминается конструкция простейших проективных моделей многообразий с пучком поверхностей дель Пеццо степени 2 , исключаются два упомянутых случая, приводятся условия общности положения для оставшихся трех случаев и показывается, что они удовлетворяются для общих многообразий, и, наконец, формулируется основной результат. В третьем содержится начало доказательства (общее в практике применения метода максимальных особенностей к расслоенным многообразиям) и исключаются простейшие случаи бесконечно близких

Работа вьполнена при поддержке Российского фонда фундаментальных исследований (гранты № № 02-01-00441, 00-15-96085), ффонда NWO-RFBR (грант № 047-008-005) и фонда INTAS (гранты № № 00-0269, 00-0259).

(C) М. М. Гриненко 2003 
особенностей. Основная и технически наиболее насышенная часть работы составляет четвертьй параграф. Пятый завершает доказательство основного утверждения.

Большая часть данной работы была написана в течение визита автора в Korea Institute for Advanced Study (Сеул, Корея). Автор признателен сотрудникам и персоналу института за поддержку и комфортные условия для работы.

\section{§1. Граф максимальной особенности и коэффициенты в неравенстве Нётера-Фано}

Понятие максимальной особенности линейной системы (грубо говоря, базисного подмножества высокой кратности) является ключевым для метода, используемого в этой и аналогичных ей статьях. Строгое определение можно найти в [4] или [3], здесь мы лишш неформально напомним, что это такое. Пусть $\mathscr{D} \subset\left|n\left(-K_{V}\right)\right|$

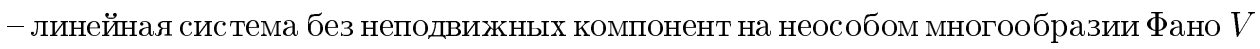
(для Фано-расслоенных пространств полагаем $\mathscr{D} \subset\left|n\left(-K_{V}\right)+A\right|$, где $A-$ поднятьй с базы эффективньй дивизор). В данном случае (рациональное) число $n$ называется порогом канонического присоединения. Предположим, что задано нормирование $\nu$ поля функций на $V$, и пусть $\nu(\mathscr{D})$ и $\delta$ - кратности соответственно нашей линейной системы и канонического дивизора относительно этого нормирования. Тогда говорят, что $\nu$ задает максимальную особенность $\mathscr{D}$, если выполнено неравенство Нётера-Фано

$$
\nu(\mathscr{D})>n \delta
$$

Мы предполагаем, что это нормирование геометрическое, т.е. реализуется цепочкой раздутий $\varphi_{i}: V_{i} \rightarrow V_{i-1}, 1 \leqslant i \leqslant N, V_{0}=V$, с неособыми центрами $B_{i-1} \subset V_{i-1}$ и исключительными дивизорами $E_{i} \subset V_{i}$, причем $B_{i} \subset E_{i}$ и $\varphi_{i}\left(B_{i}\right)=B_{i-1}$ (т.е. раздутиями с доминирующими центрами).

Таким образом, в трехмерном случае можно полагать, что $B_{0}, \ldots, B_{L-1}$ - точки, $B_{L}, \ldots, B_{N-1}-$ кривые. Пусть $\nu_{i}=\operatorname{mult}_{B_{i-1}} \mathscr{D}^{i-1}$ (всюду далее верхние индексы обозначают собственный прообраз на соответствующем этаже цепочки раздутий); положим $\delta_{i}=2$, если $i<L$ (т.е. $\varphi_{i}$ - раздутие точки), и $\delta_{i}=1$ для $i \geqslant L$ (соответствует раздутиям кривых). Тогда сушествуют числа $r_{1}, \ldots, r_{N}$ такие, что

$$
\begin{aligned}
\nu(\mathscr{D}) & =\sum_{i=1}^{N} r_{i} \nu_{i}, \\
\delta & =\sum_{i=1}^{N} r_{i} \delta_{i}
\end{aligned}
$$

и неравенство Нётера-Фано перепишется в виде

$$
\sum_{i=1}^{N} r_{i} \nu_{i}>2 n \Sigma_{0}+n \Sigma_{1}
$$

где $\Sigma_{0}=\sum_{i=1}^{L} r_{i}$ и $\Sigma_{1}=\sum_{i=L+1}^{N} r_{i}$. 
Для точного определения чисел $r_{i}$ вводится понятие графа Г максимальной особенности. Это ориентированный граф, состоящий из $N$ вершин $1, \ldots, N$, и при $j>i$ сушествует стрелка $j \rightarrow i$, если $B_{j-1} \subset E_{i}^{j-1}$. Для $j>i$ определим числа $r_{j i}$ как количество путей в графе из вершины $j$ в вершину $i ; r_{i i}=1$ по определению. Положим $r_{i}=r_{N i}$. Легко заметить, что для таким образом определенных $r_{i}$ приведенные выше разложения $\nu(\mathscr{D})$ и $\delta$ верны.

Основное (и легко доказываемое) свойство чисел $r_{i}\left(\right.$ и $\left.r_{j i}\right)$ следующее:

$$
r_{i}=\sum_{\substack{j \rightarrow i \\ i<j \leqslant N}} r_{j}
$$

В частности, $r_{1} \geqslant r_{2} \geqslant \cdots \geqslant r_{N}$.

Заметим, что $\nu_{1} \geqslant \nu_{2} \geqslant \cdots \geqslant \nu_{N}$, так что если все центры раздутий - точки, то $\nu_{1}>2 n$, а если - кривые, то $\nu_{1}>n$, и тогда из неравенства Нётера-Фано следует, что $\mathscr{D}$ имеет максимальную особенность уже на первом этаже (она реализуется одним раздутием соответственно точки или кривой). Обычно в практике метода максимальных особенностей такие случаи либо легко исключаются, либо удается “открутить” максимальную особенность при помощи бирационального автоморфизма и тем самым понизить порог канонического присоединения (грубо говоря, степень линейной системы). Поэтому самые большие проблемы связаны со случаями бесконечно близких особенностей, т.е. когда $0<L<N-1$ (вначале раздуваются $L$ точек, затем $-N-L$ кривых).

Заметим, что всегда можно считать $n<\nu_{N} \leqslant \cdots \leqslant \nu_{1} \leqslant 2 n$. Действительно, если $\nu_{i}>2 n$, то $\nu_{1}>2 n=n \delta_{1}$, так что максимальная особенность реализуется на исходном многообразии (на самом деле это не бесконечно близкий случай). Если же $\nu_{i} \geqslant n$, то, очевидно, максимальная особенность уже реализуется где-то ниже $i$-го этажа, так что нет смысла подниматься выше.

Существуют довольно сильные ограничения на структуру графа максимальной особенности. Важнейшие сформулированы в следуюшем предложении.

ПРЕДЛОЖЕНИЕ 1.1. Пусть Г - әраф бесконечно близкой максимальной особенности линейной системы $\mathscr{D}$ без неподвижсных компонент на трехмерном гладком многообразии $V$. Тогда выполнены следующие свойства:

1) если существует стрелка $j \rightarrow i, j>i$, то существуют стрелки $l \rightarrow i$ для всех $j>l>i$

2) $B_{L}-$ прямая на $E_{L} \cong \mathbb{P}^{2}$, кривие $B_{L+1}, \ldots, B_{N-1}$ - сечения соответствующих исключительных дивизоров, являющихся линейчатыми поверхностями;

3) для $L-1 \leqslant i<j \leqslant N$ стрелка $j \rightarrow i$ существует в том и только том случае, если $j=i+1$;

4) кривая $B_{L+1}$ не пересекает $E_{L}^{L+1}$.

ДокАЗАТЕЛьСТво. Почти все это показано в [5], но для удобства мы повторим аргументы. Первое утверждение прямо следует из определения “стрелки” и того, 
что нормирование разрешается цепочкой раздутий с доминируюшими центрами, т.е. если $B_{j-1} \subset E_{i}^{j-1}$, то тем более $B_{l} \subset E_{i}^{l-1}$. Далее, пусть $\operatorname{deg} B_{L}=m>1$, тогда для дивизора $D^{L} \in \mathscr{D}^{L}$ и обшей прямой $l \subset E_{L} \cong \mathbb{P}^{2}$ имеем $D^{L} \circ l=\nu_{L} \geqslant$ $m \nu_{L+1}>2 n$, что противоречит условию $n<\nu_{i} \leqslant 2 n$ для всех $i$. Аналогично для более высоких этажей: если $B_{i} \subset E_{i}, i>L$, есть $m$-кратное (неприводимое) сечение линейчатой поверхности $E_{i}$, то для ее общего слоя $l$ получаем $D^{i} \circ=\nu_{i} \geqslant$ $m \nu_{i+1}$, откуда $m=1$. Это доказывает второй пункт.

Третье утверждение: пусть существует стрелка $j \rightarrow i, j>i+1$. Тогда существует стрелка $i+2 \rightarrow i$, т.е. $B_{i+1}=E_{i}^{i+1} \cap E_{i+1}$. Предположим сначала, что $i>L$. Тогда, если $l$ - собственный прообраз слоя $E_{i}$ на $(i+1)$-м этаже, имеем $D^{i+1} \circ l=\nu_{i}-\nu_{i+1} \geqslant \nu_{i+2}$, т.е. $\nu_{i}>2 n$. В случае $i=L$ в качестве $l$ можно взять собственный прообраз общей прямой на $E_{L}$, а при $i=L-1$ - собственный прообраз общей прямой на $E_{L-1}$, проходящей через точку $B_{L-1}$.

Четвертое утверждение доказывается аналогично: если $B_{L+1}$ пересекает $E_{L}^{L+1}$ в какой-то точке, то на $E_{L} \cong E_{L}^{L+1}$ есть целый пучок прямых, проходящих через эту точку, так что для общей такой прямой $l$ имеем $D^{L+1} \circ l=\nu_{L}-\nu_{L+1} \geqslant \nu_{L+2}$, т.е. $\nu_{L}>2 n$. Заметим, что в этих утверждениях использовался тот факт, что $\mathscr{D}$ не имеет базисных компонент. Предложение доказано.

ЗАмЕчАниЕ 1.2. Напрашивается следуюшее усилениеп. 3) в предыдушем предложении: вообще нет стрелок с "верхних" этажей (где раздуваются кривые) на "нижние" (где точки) (кроме, конечно, $L+1 \rightarrow L$ ). Однако доказательство отсутствует, и, более того, это просто может оказаться неверным. Однако нечто в этом духе можно допустить. Напомним, что в методе максимальных особенностей при выводе квадратичного неравенства [4; теорема 7.1] - основного инструмента исключения бесконечно близких особенностей - единственное, что требовалось от коэффициентов $r_{i}$, это быть совместимыми со структурой графа, т.е. чтобы выполнялось неравенство

$$
r_{i} \geqslant \sum_{j \rightarrow i} r_{j} .
$$

Теперь предположим, что $r_{i}$ вместо (1.2) определены формулой

$$
r_{i}=\sum_{\substack{j \rightarrow i \\ i<j \leqslant L}} r_{j}
$$

Тогда, как легко увидеть, указанное требование на $r_{i}$ выполнено. Кроме того, неравенство Нётера-Фано также не нарушается. Действительно, по новой формуле $r_{i}$ при $i \geqslant L-1$ остаются те же, а при $i<L-1$ могут лишь уменьшиться (с сохранением упомянутого требования), так что ввиду того, что $\nu_{i} \leqslant 2 n$, неравенство (1.1) сохранится. Таким образом, сохранится и вся схема рассуждений метода максимальных особенностей. Заметим, что новое определение коэффициентов как раз соответствует графу, в котором нет стрелок с “верхних" этажей на "нижние”.

Всюду далее в тексте коэффициенты $r_{i}$ будут считаться определенньми формулой (1.3). Заметим еще, что $r_{L-1}=r_{L}=\cdots=r_{N}=1$ по п. 3) предложения 1.1, однако, используя в дальнейшем это свойство, для удобства понимания мы не будем заменять в формулах эти коэффициенты на “1”. 


\section{§2. Основной результат}

В данной работе исследуются неособые многообразия $\rho: V \rightarrow \mathbb{P}^{1}$, расслоенные на поверхности дель Пецю степени 2 над $\mathbb{P}^{1}$. Все эти многообразия предполагаются принадлежащими категории расслоений Мори. В частности, слой над общей схемной точкой базы - неособая минимальная поверхность дель Пеццо степени 2 (над полем функций прямой), все слои неприводимы и приведены (последнее следует из неособости). Таким образом,

$$
\operatorname{Pic}(V)=\mathbb{Z}\left[-K_{V}\right] \oplus \mathbb{Z}[F]
$$

где $F$ - класс слоя.

Воспроизведем геометрическую конструкцию многообразий указанного типа. Поскольку все слои являются приведенными горенштейновыми поверхностями дель Пещо степени 2, то, как хорошо известно, антиканоническая линейная система на таких поверхностях свободна от базисных точек и определяет конечный морфизм степени 2 на плоскость с ветвлением вдоль квартики. Таким образом, линейная система $\left|-K_{V}+l F\right|$ на $V$ не имеет базисных точек при $l \gg 0$ и задает конечный морфизм $\varphi: V \rightarrow X$ степени 2 на многообразие $X$, которое может быть получено следуюшим образом. Заметим, что $\rho_{*} \mathscr{O}\left(-K_{V}+l F\right)=\mathscr{E}$ есть локально свободный пучок ранга 3 , и мы можем полагать $l$ выбранньм так, что $\mathscr{E}=\mathscr{O} \oplus \mathscr{O}\left(n_{1}\right) \oplus \mathscr{O}\left(n_{2}\right), 0 \leqslant n_{1} \leqslant n_{2} . \quad$ Тогда $X=\operatorname{Proj}_{\mathbb{P}^{1}} \mathscr{E} . \quad$ Относительно естественной проекции $\pi: X \rightarrow \mathbb{P}^{1}$ многообразие $X$ есть расслоение на плоскости, согласованное с расслоением $\rho: V \rightarrow \mathbb{P}^{1}$ посредством морфизма $\varphi$, т.е. $\rho=\pi \circ \varphi$.

Далее, очевидно, что

$$
\operatorname{Pic}(X)=\mathbb{Z}[M] \oplus \mathbb{Z}[L],
$$

где $M$ - класс тавтологического пучка $\left(\rho_{*} \mathscr{O}(M)=\mathscr{E}\right), L$ - класс слоя проекции $\pi$. Пусть $t_{0}$ - сечение $X$, соответствуюшее сюръективному отображению $\mathscr{E} \rightarrow \mathscr{O}_{\mathbb{P} 1} \rightarrow 0$ (или, что то же самое, удовлетворяющее условию $M \circ t_{0}=0$ ), и $l-$ класс прямой в слое отображения $\pi$. Морфизм $\varphi$ разветвлен над неособым дивизором $R$ на $X$, расслоенным на квартики над $\mathbb{P}^{1}$, т.е. имеющим класс $R \sim 4 M+2 a L$. Заметим, что с точностью до деформаций числа $n_{1}, n_{2}$ и $a$ полностью определяют расслоение $V$.

Обозначим $b=n_{1}+n_{2}, H=\varphi^{*}(M), s_{0}=\frac{1}{2} \varphi^{*}\left(t_{0}\right)$ и $f=\frac{1}{2} \varphi^{*}(l)$. Очевидно, $F=\varphi^{*}(L)$. Нетрудно видеть, что $M^{2}=t_{0}+b l, M^{3}=b, M \circ l=L \circ t_{0}=1$, $M \circ t_{0}=L \circ l=0, K_{X} \sim-3 M+(b-2) L, H^{2}=2 s_{0}+2 b f, H^{3}=2 b, H \circ f=F \circ s_{0}=1$, $H \circ s_{0}=F \circ f=0, K_{V} \sim-H+(a+b-2) F, K_{V}^{2} \sim 2 s_{0}+(8-4 a-2 b) f$.

Отметим, что линейные системы $\left|M-n_{2} L\right|$ и $\left|H-n_{2} F\right|$ непусты и в дальнейшем их общие дивизоры (а в случаях, разбираемых ниже, это единственные дивизоры) обозначаются $G$ и $G_{V}$ соответственно. Эти своего рода "минимально закрученные" над базой дивизоры играют важную роль в геометрии многообразий $X$ и $V$, примерно такую же, как исключительные сечения в линейчатых поверхностях.

Напомним, что в работе [1] следующие случаи были перечислены, но не рассмотрены: 


$$
\begin{gathered}
\text { при } b+2 a=2 \\
n_{1}=2, n_{2}=4, a=-2 ; \\
n_{1}=2, n_{2}=6, a=-3 ; \\
n_{1}=2, n_{2}=8, a=-4 ; \\
n_{1}=0, n_{2}=2, a=0 ; \\
\text { при } b+2 a=1 \\
n_{1}=1, n_{2}=4, a=-2 .
\end{gathered}
$$

Первый результат данной статьи - это

ПРЕДЛОЖЕНИЕ 2.1. Случаи $n_{1}=2, n_{2}=8, a=-4(n p u b+2 a=2) u n_{1}=1$, $n_{2}=4, a=-2($ при $b+2 a=1)$ не реализуются.

ДокАЗАТЕЛьство. Заметим, что и в том, и в другом случаях $X$ содержит единственное сечение класса $t_{0}$ и

$$
\left.R\right|_{G} \sim 4 t_{0}
$$

Таким образом, $G_{V}=\varphi^{*}(G)$ распадается на две линейчатые поверхности (касающиеся друг друга вдоль сечения класса $s_{0}$ ), так что, очевидно, слой над общей схемной точкой содержит две $(-1)$-кривые, т.е. не минимален. Таким образом, $V / \mathbb{P}^{1}$ не лежит в категории расслоений Мори (относительное число Пикара больше единицы). Предложение доказано.

Всюду далее в тексте ссылки на оставшиеся случаи многообразий устроены следующим образом:

а) $\mathscr{M}_{1}$ соответствует $n_{1}=2, n_{2}=6, a=-3$;

b) $\mathscr{M}_{2}$ соответствует $n_{1}=2, n_{2}=4, a=-2$;

c) $\mathscr{M}_{3}$ соответствует $n_{1}=0, n_{2}=2, a=0$.

Ниже будет доказано, что многообразия этих типов являются бирационально жесткими, если выполнены условия общности $\mathscr{C} \mathscr{M}_{1}, \mathscr{C} \mathscr{M}_{2}$ и $\mathscr{C} \mathscr{M}_{3}$ для типов $\mathscr{M}_{1}$, $\mathscr{M}_{2}$ и $\mathscr{M}_{3}$ соответственно. Сформулируем эти условия.

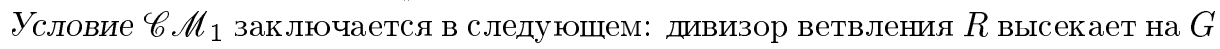
(а это линейчатая поверхность типа $\mathbb{F}_{2}$ ) тройное исключительное сечение и сечение, не пересекающее исключительное.

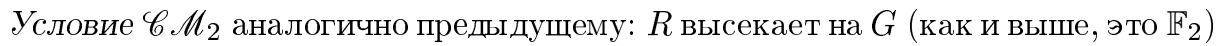
двойное исключительное сечение и бисечение (возможно, приводимое), не пересекающее исключительное.

Условие $\mathscr{C} \mathscr{M}_{3}$ содержит два требования. Первое требование: $V$ имеет общие вырождения, т.е. не более одной особой точки типа $\mathbb{A}_{1}$ в слое, или, что то же самое, $R$ имеет простейшие вырождения. Далее, нетрудно видеть, что $G \cong \mathbb{P}^{1} \times \mathbb{P}^{1}$ заметается кривыми класса $t_{0}$, причем $R$ высекает на $G$ в точности 4 различные сечения этого класса (последнее следует из неособости $R$ и $G$ : достаточно раздуть кривую их пересечения и убедиться, что собственные прообразы высекают на исключительном дивизоре непересекающиеся сечения). Любой слой $X$ (а это плоскости) указанные четыре сечения пересекают в 4 различных точках. Второе требование состоит в том, что через эти точки не проходя $m$ четырехкратные касательные к дивизору ветвления (но если одна из четырех точек совпадает с особой точкой ограничения дивизора ветвления на слой, то требование на такую точку 
снимается). На $V$ это требование выглядит следующим образом: те антиканонические кривые в слоях, которые распадаются на пару касающихся друг друга прямых, не пересекают ни одно из четырех сечений класса $s_{0}$, что лежат над указанными сечениями класса $t_{0}$ (кроме случая, когда особая точка такой антиканонической кривой совпадает с особой точкой слоя).

Лемма 2.2. Условия $\mathscr{C} \mathscr{M}_{1}, \mathscr{C} \mathscr{M}_{2}$ и $\mathscr{C} \mathscr{M}_{3}$ выполняются для общих многообразий соответствующих типов.

ДокАЗАТЕЛьство. Начнем с условия $\mathscr{C} \mathscr{M}_{1}$. В данном случае мы имеем $G \sim$ $M-6 L$ и $R \sim 4 M-6 L, G$ есть $\mathbb{F}_{2}$-поверхность, классы исключительного сечения и слоя которой обозначим так же, как и в $X: t_{0}$ и $l$. Таким образом,

$$
\left.R\right|_{G} \sim 4 t_{0}+2 l
$$

Утверждение леммы теперь вытекает из точной тройки

$$
0 \rightarrow \mathscr{O}_{V}(R-G) \rightarrow \mathscr{O}_{V}(R) \rightarrow \mathscr{O}_{G}(R) \rightarrow 0
$$

Действительно, $h^{1}\left(\mathscr{O}_{V}(R-G)\right)=h^{1}(3 M)=0$, так что отображение $H^{0}\left(\mathscr{O}_{V}(R)\right) \rightarrow$ $H^{0}\left(\mathscr{O}_{G}(R)\right)$ сюръективно. Осталось лиш заметить, что общий элемент линейной системы $\left|4 t_{0}+2 l\right|$ на $G$ есть тройное исключительное сечение плюс сечение, не пересекающее исключительное (т.е. класса $\left.t_{0}+2 l\right)$. Условие $\mathscr{C} \mathscr{M}_{2}$ доказывается аналогично.

Чтобы убедиться в том, что условие $\mathscr{C} \mathscr{M}_{3}$ выполнено для обшего многообразия типа $\mathscr{M}_{3}$, рассмотрим следуюшую конструкцию. Линейная система $|M|$ не имеет базисных точек на $X$ и задает бирациональный морфизм $X \rightarrow Q$ на квадрику ранга 3 в $\mathbb{P}^{4}$ (т.е. $Q$ - это одномерное семейство плоскостей, проходящих через некоторую прямую $l \subset \mathbb{P}^{4}$ и параметризованных коникой, если рассматривать проекцию $\mathbb{P}^{4} \rightarrow \mathbb{P}^{2}$ с центром в $l$ ). Указанный бирациональный морфизм стяпивает $G \sim \mathbb{P}^{1} \times \mathbb{P}^{1}$ на $l$. Образ $R$ при этом морфизме - дивизор $R^{\prime}$ на $Q$, высекаемый квартикой $S \subset \mathbb{P}^{4}$. Заметим, что $S$ пересекает $l$ в четырех различных точках. Обратно, по паре $(Q, S)$ в обшем положении восстанавливается пара $(X, R)$ с требуемыми свойствами.

Заметим, что через обшую точку неособой квартики $S \subset \mathbb{P}^{4}$ проходит 6 четверных касательных. Далее все просто: рассмотрим общую прямую $l$, пересекающую $S$ в 4 общих точках, и возьмем проекцию $\mathbb{P}^{4} \rightarrow \mathbb{P}^{2}$ из $l$. Четверные касательные к $S$ через точки $l \cap S$ определяют 24 точки на плоскости при этой проекции, сама $S$ представляется расслоенной на квартики с приведенной дискриминантной кривой $s \subset \mathbb{P}^{2}$. Выбрав конику $q$ в общем положении с $s$, не проходяшую через указанные 24 точки, мы по ней построим квадрику $Q$ ранга 3 такую, что пара $(Q, S)$ (и построенная по ней $(X, R))$ будет удовлетворять всем требованиям условия $\mathscr{C} \mathscr{M}_{3}$. Лемма доказана.

Теорема 2.3. Многообразия $\mathscr{M}_{1}, \mathscr{M}_{2}$ и $\mathscr{M}_{3}$ бирационально жестки, если выполнены условия $\mathscr{C} \mathscr{M}_{1}, \mathscr{C} \mathscr{M}_{2}$ и $\mathscr{C} \mathscr{M}_{3}$ соответственно. 
ЗАмечАниЕ 2.4. Автор полагает, что приведенные условия общности не являются необходимыми. Как это часто бывает в практике метода максимальных особенностей, поначалу доказывается результат для общих многообразий, затем каким-то образом избавляются от условий общности (или придумывают новое доказательство).

ЗАмЕчАнИЕ 2.5. Гипотетический критерий жесткости для неособых пучков поверхностей дель Пецщо, сформулированный автором в [1], работает и для перечисленных вьше многообразий: $V / \mathbb{P}^{1}$ жестко, если и только если линейные системы вида $\left|n\left(-K_{V}\right)-F\right|$ либо пусты, либо имеют неподвижную компоненту. В нашем случае линейные системы такого вида непусты, но имеют неподвижную компоненту $G_{V}$.

Оставшаяся часть статьи содержит доказательство сформулированной теоремы.

\section{§3. Начало доказательства теоремы 2.3}

Пусть $\chi: V \rightarrow V^{\prime}-$ бирациональное отображение многообразия типа $\mathscr{M}_{1}, \mathscr{M}_{2}$ или $\mathscr{M}_{3}$ на Мори-расслоение $\rho^{\prime}: V^{\prime} \rightarrow S^{\prime}, \mathscr{D}^{\prime}=\left|n^{\prime}\left(-K_{V^{\prime}}\right)+A^{\prime}\right|$ - линейная система без базисных точек и $A^{\prime}$-поднятый с базы эффективный дивизор.

Рассмотрим собственный прообраз $\mathscr{D}=\chi_{*}^{-1} \mathscr{D}^{\prime}$ линейной системы $\mathscr{D}^{\prime}$. Тогда $\mathscr{D}$ не имеет базисных компонент, и легко проверить, что $\mathscr{D} \subset\left|n\left(-K_{V}\right)+m F\right|$ для некоторого $m \geqslant 0$ (если $m<0$, то $\mathscr{D}$ обязательно имела бы $G_{V}$ в качестве неподвижной компоненты, так как пересечения с кривыми класса $2 s_{0}+2 n_{1} f$, покрываюшими $G_{V}$, будут отрицательны). Хорошо известно [6; предложение 4.2], что $n \geqslant n^{\prime}$ в данной ситуации, и если $n=n^{\prime}$, то $\chi$ есть отображение над базой (т.е. то, что нам нужно для доказательства бирациональной жесткости).

Предположим, что $n>n^{\prime}$. Тогда $\mathscr{D}$ имеет максимальную особенность. Пусть центр максимальной особенности на $V$ - кривая. Тогда эта кривая обязательно является сечением, не лежащим на дивизоре ветвления двойного накрытия $\varphi: V \rightarrow X$, и при помощи инволюций Бертини все такие максимальные особенности могут быть “откручены" [2; 3$]$. Таким образом, можно предполагать, что центры всех максимальных особенностей на $V$ - только точки. В этих условиях доказывается, что все такие максимальные особенности обязательно бесконечно близкие, и существует оценка сверху на число $m$ [2; предложение 2.1].

Точнее, утверждается следующее. Существует конечное множество $\mathscr{T}$ исключительных дивизоров над $V$, стягиваемых в точки, такое, что для соответствующего множества дискретных нормирований $\left\{\nu_{T}: T \in \mathscr{T}\right\}$ выполнено неравенство

$$
m<\sum_{t \in \mathbb{P}^{1}} \max _{\substack{T \in \mathscr{T}: \\ \text { Center } \nu_{T} \in F_{t}}} \frac{e_{T}}{\nu_{T}\left(F_{t}\right)}
$$

где $F_{t}$ - слой над точкой $t \in \mathbb{P}^{1}, e_{T}=\nu_{T}(\mathscr{D})-n \delta_{T}, \delta_{T}-$ каноническая кратность (дискрепантность) по отношению к нормированию $\nu_{T}$. Через $M$ обозначим количество всех различных слоев $V$, содержащих максимальные особенности (т.е. количество слагаемых в (3.1)). 
Пусть $D_{1}$ и $D_{2}$ - общие элементы линейной системы $\mathscr{D}$. Так как $\mathscr{D}$ не имеет неподвижных компонент, $D_{1} \circ D_{2}$ будет эффективным 1-циклом, который может быть представлен в виде суммы эффективных горизонтальных и вертикальных циклов следуюшим образом:

$$
D_{1} \circ D_{2}=Z_{0}+Z^{h}+Z^{v}, \quad Z^{v}=\sum_{t \in \mathbb{P}^{1}} Z_{t}^{v} .
$$

Здесь вертикальные циклы $Z_{t}^{v}$ - эффективные кривые, лежащие в слое над точкой $t$, горизонтальный цикл $Z_{0} \sim \alpha s_{0}$ составлен из сечений класса $s_{0}$ и $Z^{h}$ - горизонтальный цикл, носитель которого не содержит сечений класса $s_{0}$. Необходимо отметить, что на многообразиях, изучаемых в данной статье, любая неприводимая приведенная кривая, класс которой кратен $s_{0}$, обязательно является сечением (и тогда ее класс есть в точности $s_{0}$ ), поэтому предложенное разбиение горизонтальных циклов на сумму двух корректно.

В дальнейшем нам нужны будут оценки на кратности ("mult") циклов в некоторой точке многообразия $V$. Легко видеть, что такая кратность не превосходит степени ("deg") цикла, если под степенью для горизонтальных циклов понимать пересечение с классом слоя (т.е. $F$ ), а для вертикальных - с классом $H$.

Заметим, что

$$
D_{1} \circ D_{2} \sim 2 n^{2} s_{0}+\left(4 n^{2}+4 m n\right) f
$$

откуда мы можем немедленно получить оценки на кратности циклов в некоторой (произвольной) точке $B \in V$ :

$$
\begin{gathered}
\operatorname{mult}_{B} Z_{0} \leqslant \operatorname{deg} Z_{0}=\alpha, \quad \operatorname{mult}_{B} Z^{h} \leqslant \operatorname{deg} Z^{h}=2 n^{2}-\alpha \\
\operatorname{mult}_{B}\left(\sum_{t} Z_{t}^{v}\right) \leqslant \operatorname{deg}\left(\sum_{t} Z_{t}^{v}\right)=\operatorname{deg} \sum_{t} Z_{t}^{v} \leqslant 4 n^{2}+4 m n
\end{gathered}
$$

Из последнего неравенства и (3.1) мы получаем условие на сверхмаксимальную особенность (аналогично следствию 4.1 в [2], но с учетом числа $M$ слоев с максимальными особенностями): существуют точка $t_{0} \in \mathbb{P}^{1}$ и дискретное нормирование $\nu_{T}$ с центром в точке $B_{0} \in F_{t_{0}}$ такие, что

$$
\operatorname{mult}_{B_{0}} Z_{t_{0}}^{v} \leqslant \operatorname{deg} Z_{t_{0}}^{v}<\frac{4 n^{2}}{M}+\frac{4 n e_{T}}{\nu_{T}\left(F_{t_{0}}\right)} .
$$

Покажем, что обязательно $M=1$.

Возьмем разрешение указанного нормирования $\nu_{T}$, как в $\S 1$, для этого разрешения - граф $\Gamma$, по которому определяются числа $N, L, r_{i}, \Sigma_{0}$ и $\Sigma_{1}$ (в обозначениях $\S 1)$. Кроме того, определим

$$
k=\max \left\{1 \leqslant i \leqslant L: B_{i-1} \in F_{t_{0}}^{i-1}\right\}
$$

(напомним, верхние индексы обозначают собственный прообраз на соответствующем этаже цепочки раздутий $), k$, неформально говоря, показывает, как долго центры раздутий будут “сидеть” на собственном прообразе слоя, и непосредственно 
измеряет тот “довесок", которьй превращает максимальную особенность в сверхмаксимальную. Заметим, что точка $B_{0}$ (а значит, и $B_{1}, \ldots, B_{L-1}$ ) обязательно гладкая на $F_{t_{0}}$, откуда

$$
\Sigma_{0}^{\prime} \stackrel{\text { def }}{=} \sum_{i=1}^{k} r_{i} \leqslant \nu_{T}\left(F_{t_{0}}\right)
$$

Отметим, что $\Sigma_{0}^{\prime} \leqslant \Sigma_{0}$.

Согласно общей теории метода максимальных особенностей [2; предложение 4.2], [4; следствие 7.2] мы имеем так называемое квадратичное неравенство:

$$
\Sigma_{0} \text { mult }_{B_{0}} Z_{0}+\Sigma_{0} \text { mult }_{B_{0}} Z^{h}+\Sigma_{0}^{\prime} \text { mult }_{B_{0}} Z^{v} \geqslant \frac{\left(2 n \Sigma_{0}+n \Sigma_{1}+e\right)^{2}}{\Sigma_{0}+\Sigma_{1}}
$$

Предположим, что $M \geqslant 2$. Тогда

$$
\operatorname{mult}_{B_{0}} Z_{t_{0}}^{v} \leqslant \operatorname{deg} Z_{t_{0}}^{v}<2 n^{2}+\frac{4 n e}{\Sigma_{0}^{\prime}}
$$

и мы получаем противоречие:

$$
\left(\alpha \Sigma_{0}+\left(2 n^{2}-\alpha\right) \Sigma_{0}+2 n^{2} \Sigma_{0}+4 n e\right)\left(\Sigma_{0}+\Sigma_{1}\right)>\left(2 n \Sigma_{0}+n \Sigma_{1}+e\right)^{2} .
$$

Таким образом, все максимальные особенности сосредоточены в одном слое, т.е. $M=1$.

ЛЕмма 3.1. Предположим, что D имеет только бесконечно близкие максимальные особенности, центры которых сосредоточены в одном слое (скажем, над точкой $\left.t_{0} \in \mathbb{P}^{1}\right)$. Тогда хотя бъ для одного из нормирований, реализующих эти максимальные особенности (скажем, $\left.\nu_{T}\right)$, выполнено усиленное неравенство Нётера-Фано

$$
\nu_{T}(\mathscr{D})>n \delta_{T}+m \nu_{T}\left(F_{t_{0}}\right)
$$

или в терминах графа максимальной особенности (§1)

$$
\sum_{i=1}^{N} r_{i} \nu_{i}>2 n \Sigma_{0}+n \Sigma_{1}+m \Sigma_{0}^{\prime}
$$

əде, как и выцие, $\nu_{i}=\operatorname{mult}_{B_{i-1}} \mathscr{D}^{i-1}, \Sigma_{0}^{\prime}=\sum_{i=1}^{k} r_{i}$. 
ДокАЗАТЕЛЬСтво. Аргументы почти дословно повторяют вывод теоремы о сушествовании максимальной особенности при $n>n^{\prime}$ (см., например, [2; предложение 2.1]). Выберем разрешение $V \leftarrow W \rightarrow V^{\prime}$ бирационального отображения $\chi: V \rightarrow V^{\prime}$ (скажем, по Хиронаке). Пусть $\mathscr{D}_{W}-$ собственньй прообраз линейной системы $\mathscr{D}$ (или $\left.\mathscr{D}^{\prime}\right)$ на $W$. Тогда пара $n K_{W}+\mathscr{D}_{W}$ не имеет эфффективных представителей. Осталось учесть, что $n K_{V}+\mathscr{D} \sim m F$ и центры всех максимальных особенностей лежат в одном слое. Для вывода второго неравенства достаточно заметить, что $\nu_{T}\left(F_{t_{0}}\right) \geqslant \Sigma_{0}^{\prime}$. Лемма доказана.

Итак, на данный момент мы имеем следующую ситуацию. Сушествует дискретное нормирование $\nu_{T}$ с центром в точке $B_{0} \in V$, являющейся неособой точкой некоторого слоя, который в дальнейшем будет просто обозначаться $F$; для линейной системы $\mathscr{D}$ выполнено уточненное неравенство Нётера-Фано из леммы 3.1 (относительно $\nu_{T}$ ); определены числа $k, L, N, r_{i}$ (последние - с учетом замечания 1.2 и формулы (1.3)).

Далее, квадратичное неравенство может быть записано в виде

$$
\widetilde{\Sigma}_{0} \text { mult }_{B_{0}} Z_{0}+\Sigma_{0} \text { mult }_{B_{0}} Z^{h}+\Sigma_{0}^{\prime} \text { mult }_{B_{0}} Z^{v} \geqslant \sum_{i=1}^{N} r_{i} \nu_{i}^{2}>\varphi(\Gamma),
$$

где

$$
\varphi(\Gamma)=\frac{\left(2 n \Sigma_{0}+n \Sigma_{1}+m \Sigma_{0}^{\prime}\right)^{2}}{\Sigma_{0}+\Sigma_{1}}
$$

зависит только от структуры графра $\Gamma$, а $\widetilde{\Sigma}_{0}$ определяется следующим образом. Пусть $C \subset Z_{0}$ - сечение класса $s_{0}$, проходящее через точку $B_{0}$ (а если таких нет, то полагаем $\widetilde{\Sigma}=0$ ), и

$$
l=\max \left\{1 \leqslant i \leqslant L: B_{i-1} \in C^{i-1}\right\}
$$

Тогда $\widetilde{\Sigma}_{0}=\sum_{i=1}^{l} r_{i}$.

В оставшейся части статьи будет показано, что на самом деле описанная ситуация приводит к противоречию, откуда неизбежно $n=n^{\prime}$ и отображение $\chi$ проводится через базу, что как раз и означает жесткость рассматриваемых многообразий. Доказательство будет сведено к разбору возможных частных случаев. В этом параграфе показывается, что через точку $B_{0}$ обязательно должно проходить сечение класса $s_{0}$, что случай $L>k=1$ невозможен, а также выводится уточненное квадратичное неравенство и некоторые прямые следствия из него.

Но прежде сделаем два замечания о горизонтальных циклах на $V$. Во-первых, в случаях $\mathscr{M}_{1}$ и $\mathscr{M}_{2}$ на многообразии имеется единственное сечение класса $s_{0}$, а в $\mathscr{M}_{3}$ такие сечения заметают дивизор $G_{V}$, причем различные сечения не пересекаются (они лежат над одним из двух семейств образующих поверхности $G \cong \mathbb{P}^{1} \times \mathbb{P}^{1}$, имеющих класс $\left.t_{0}\right)$ и четыре из них лежат на дивизоре ветвления $R_{V}$ морфизма $\varphi: V \rightarrow X$

Второе замечание состоит в следующем. Пусть $C \sim \beta s_{0}+\gamma f$ - неприводимая приведенная горизонтальная кривая на $V$, отличная от сечения класса $s_{0}$. Тогда $\gamma \geqslant \beta$ всегда, кроме ситуации, когда $V$ имеет тип $\mathscr{M}_{3}$ и $C \subset G_{V}$. Действительно, 
во всех случаях если $\beta>\gamma$, то $C \circ(H-2 F)=\gamma-2 \beta<0$, и остается лишш заметить, что $|H-2 F|$ состоит из единственного элемента $G_{V}$ в ситуации $\mathscr{M}_{3}$ и имеет базисным множеством (единственное) сечение класса $s_{0}$ в ситуациях $\mathscr{M}_{1}$ и $\mathscr{M}_{2}$.

ЛЕмма 3.2. Квадратичное неравенство может бъть переписано в виде

$$
\alpha \widetilde{\Sigma}_{0}+\left(2 n^{2}-\alpha\right) \Sigma_{0}+\left(2 n^{2}+\alpha+4 m n\right) \Sigma_{0}^{\prime}>\varphi(\Gamma) .
$$

ДоказАТЕльство. Это прямое следствие предыдущего замечания. Мы имеем

$$
D_{1} \circ D_{2} \sim 2 n^{2} s_{0}+\left(4 n^{2}+4 m n\right) f
$$

затем $Z_{0} \sim \alpha s_{0}, Z^{h} \sim\left(2 n^{2}-\alpha\right) s_{0}+\gamma f$ и $Z^{v} \sim\left(4 n^{2}+4 m n-\gamma\right) f$. В ситуациях $\mathscr{M}_{1}$ и $\mathscr{M}_{2}$, как только что было замечено, $\gamma \geqslant 2 n^{2}-\alpha$, поэтому

$$
\begin{aligned}
& \text { mult }_{B_{0}} Z_{0} \leqslant \operatorname{deg} Z_{0}=\alpha \text {, } \\
& \text { mult }_{B_{0}} Z^{h} \leqslant \operatorname{deg} Z^{h}=2 n^{2}-\alpha \text {, } \\
& \text { mult }_{B_{0}} Z^{v} \leqslant \operatorname{deg} Z^{v} \leqslant 2 n^{2}+4 m n+\alpha
\end{aligned}
$$

и осталось лишш подставить это в квадратичное неравенство.

Ситуация $\mathscr{M}_{3}$ немного сложнее. Положим $Z^{h}=Z_{1}^{h}+Z_{2}^{h}$, где

$$
\begin{array}{ll}
Z_{1}^{h}=\beta_{1} s_{0}+\gamma_{1} f, & \gamma_{1} \geqslant \beta_{1}, \\
Z_{2}^{h}=\beta_{2} s_{0}+\gamma_{2} f, & \gamma_{2}<\beta_{2}
\end{array}
$$

тогда $\beta_{1}+\beta_{2}=2 n^{2}-\alpha$ и $Z^{v} \sim\left(4 n^{2}+4 m n-\gamma_{1}-\gamma_{2}\right) f$. Как и ранее, mult $B_{0} Z_{1}^{h} \leqslant$ $\operatorname{deg} Z_{1}^{h}=\beta_{1}$, а вот степень $Z_{2}^{h}$ можно оценить пересечением с общим элементом линейной системы $|H|$, проходящим через точку $B_{0}$ (такие, легко видеть, найдутся). Таким образом, mult $B_{0} Z_{2}^{h} \leqslant Z_{2}^{h} \circ H=\gamma_{2}$. Учитывая, что

$$
\operatorname{mult}_{B_{0}} Z^{v} \leqslant 4 n^{2}+4 m n-\gamma_{1}-\gamma_{2} \leqslant 4 n^{2}+4 m n-\beta_{1}-\gamma_{2},
$$

получаем оценку сверху на левую часть квадратичного неравенства:

$$
\begin{aligned}
& \widetilde{\Sigma}_{0} \text { mult }_{B_{0}} Z_{0}+\Sigma_{0} \text { mult }_{B_{0}} Z^{h}+\Sigma_{0}^{\prime} \text { mult }_{B_{0}} Z^{v} \\
& \quad \leqslant \alpha \widetilde{\Sigma}_{0}+\left(\beta_{1}+\gamma_{2}\right) \Sigma_{0}+\left(4 n^{2}+4 m n-\beta_{1}-\gamma_{2}\right) \Sigma_{0}^{\prime} \\
& \quad \leqslant \alpha \widetilde{\Sigma}_{0}+\left(\beta_{1}+\beta_{2}\right) \Sigma_{0}+\left(4 n^{2}+4 m n-\beta_{1}-\beta_{2}\right) \Sigma_{0}^{\prime} \\
& \quad=\alpha \widetilde{\Sigma}_{0}+\left(2 n^{2}-\alpha\right) \Sigma_{0}+\left(2 n^{2}+\alpha+4 m n\right) \Sigma_{0}^{\prime},
\end{aligned}
$$

так как $\left(\beta_{2}-\gamma_{2}\right)\left(\Sigma_{0}-\Sigma_{0}^{\prime}\right) \geqslant 0$. Лемма доказана.

Лемма 3.3. Случай, когда через точку $B_{0}$ не проходит сечение класса $s_{0}$, невозможен. 
ДокАЗАТЕЛЬСТво. Пусть справедливо обратное. В такой ситуации $\widetilde{\Sigma}_{0}=0$ и тогда неравенство (3.3) дает

$$
4 n^{2} \Sigma_{0}+4 m n \Sigma_{0}^{\prime}>\varphi(\Gamma)
$$

Получаем противоречие:

$$
n^{2} \Sigma_{1}^{2}-2 m n \Sigma_{1} \Sigma_{0}^{\prime}+m^{2} \Sigma_{0}^{\prime 2}<0
$$

Лемма доказана.

Лемма 3.4. Случай $\Sigma_{0} \geqslant 2 \Sigma_{0}^{\prime}$, в частности, когда $L>k=1$ (т.е. раздуваются как минимум две точки и уже вторая "соскакивает" с собственного прообраза слоя), невозмохен.

ДокАЗАТЕЛЬСТво. Предположим противное. Так как $\widetilde{\Sigma}_{0} \leqslant \Sigma_{0}$, то по (3.3)

$$
\left(\alpha+2 n^{2}-\alpha+n^{2}+\frac{1}{2} \alpha\right) \Sigma_{0}+4 m n \Sigma_{0}^{\prime}>\varphi(\Gamma)
$$

и, поскольку $\alpha \leqslant 2 n^{2}$, получаем противоречие, как и в предыдушей лемме:

$$
4 n^{2} \Sigma_{0}+4 m n \Sigma_{0}^{\prime}>\varphi(\Gamma)
$$

Осталось лишш заметить, что если $L>k=1$, то $\Sigma_{0}^{\prime}=r_{1} \leqslant \frac{1}{2} \Sigma_{0}$ по формуле (1.3). Лемма доказана.

Таким образом, далее необходимо рассмотреть случаи, когда через точку $B_{0}$ проходит сечение класса $s_{0}$ и при этом либо $L=k$, либо $L>k \geqslant 2$. Заметим, что во всех этих ситуациях центр второго раздутия (т.е. $B_{1}$ ) “задеваее” собственный прообраз слоя на первом этаже $F^{1}$ и $\widetilde{\Sigma}_{0}=r_{1}$. Действительно, если $k \geqslant 2$, то $B_{1} \in F^{1}$ по определению числа $k$. А с другой стороны, любое сечение (в том числе класса $s_{0}$, проходящее через точку $B_{0}$ ) трансверсально пересекает слой $F$, и потому его собственный прообраз будет дизъюнктен с $F^{1}$, так что $\widetilde{\Sigma}_{0}=r_{1}$. Если же $L=k=1$, то $\widetilde{\Sigma}_{0}=r_{1}$ автоматически, а $B_{1}-$ прямая на $E_{1}$ и, очевидно, $B_{1} \cap F^{1} \neq \varnothing$.

Значит, квадратичное неравенство может быть записано как

$$
\alpha r_{1}+\left(2 n^{2}-\alpha\right) \Sigma_{0}+\left(2 n^{2}+4 m n+\alpha\right) \Sigma_{0}^{\prime}>\varphi(\Gamma)
$$

Кроме того, из факта, что центр второго раздутия всегда “задевает” собственный прообраз слоя, следует 
Лемма 3.5. Справедливо неравенство $\nu_{1}+\nu_{2} \leqslant 3 n$.

Лемма будет доказана в следующем параграфее наряду с более сильными оценками на суммы кратностей $\nu_{i}$.

Заметим, что ситуация $k=1$ ввиду леммы 3.4 возможна, только если $L=k=1$. Этот случай рассмотрим отдельно позднее, а пока все время будем предполагать $k \geqslant 2$. При выполнении этого условия можно получить некоторые полезные оценки.

Положим $x=\Sigma_{0} / \Sigma_{0}^{\prime}$. По лемме $3.41 \leqslant x<2$. Пусть $y$ такой, что $r_{1} \leqslant y \Sigma_{0}$. Так как $k \geqslant 2$, всегда можно предполагать $0<y \leqslant \frac{1}{2}$. Тогда из неравенства (3.4)

$$
\alpha x y+\left(2 n^{2}-\alpha\right) x+2 n^{2}+\alpha+4 m n>4 n^{2} x+4 m n+\frac{\left(n \Sigma_{1}-m \Sigma_{0}^{\prime}\right)^{2}}{\Sigma_{0}^{\prime}\left(\Sigma_{0}+\Sigma_{1}\right)}
$$

Обозначим

$$
\psi(\Gamma)=n^{2} \frac{\left(\Sigma_{1} / \Sigma_{0}^{\prime}-m / n\right)}{\Sigma_{0} / \Sigma_{0}^{\prime}+\Sigma_{1} / \sigma_{0}^{\prime}}
$$

Тогда

$$
\alpha>2 n^{2} \frac{(x-1+\psi(\Gamma) / 2)}{1-(1-y) x} .
$$

Предположим, что $\nu_{L+1} \leqslant \frac{13}{10} n$. Так как $\nu_{1}+\nu_{2} \leqslant 3 n$, то из неравенства Нётера-Фано (3.2) получаем, что

$$
\frac{\Sigma_{1}}{\Sigma_{0}^{\prime}}>\frac{5}{3} \frac{\Sigma_{0}}{\Sigma_{0}^{\prime}}+\frac{8}{3} \frac{m}{n}
$$

Действительно, пусть $\theta=\frac{1}{2}\left(\nu_{1}+\nu_{2}\right)$. Заметим, что $2 \theta \geqslant \nu_{1}+\nu_{i}$ и $\theta \geqslant \nu_{i}$ для всех $i \geqslant 2$. Тогда если $r_{1}=r_{2}+\cdots+r_{i}$ для некоторого $i \leqslant L$ (ввиду (1.3)), то

$$
\begin{aligned}
& r_{1} \nu_{1}+\cdots+r_{L} \nu_{L}=r_{2}\left(\nu_{1}+\nu_{2}\right)+\cdots+r_{i}\left(\nu_{1}+\nu_{i}\right)+\cdots+r_{L} \nu_{L} \\
& \quad \leqslant 2 \theta r_{2}+\cdots+2 \theta r_{i}+\theta r_{i+1}+\cdots+\theta r_{L}=\theta r_{1}+\cdots+\theta r_{L} \leqslant \frac{3}{2} \Sigma_{0}
\end{aligned}
$$

Осталось лишш подставить в (3.2) неравенство

$$
r_{L+1} \nu_{L+1}+\cdots+r_{N} \nu_{N} \leqslant \frac{13}{10} n \Sigma_{1}
$$

Итак, полученная только что оценка на $\Sigma_{1} / \Sigma_{0}^{\prime}$ влечет $\psi(\Gamma)>1$ и тогда $(3.5)$ ведет к противоречию:

$$
2 n^{2} \geqslant \alpha>2 n^{2}
$$

Таким образом, всегда $\nu_{L+1}>\frac{13}{10} n$.

Далее, положим $n \theta=\frac{1}{2}\left(\nu_{1}+\nu_{2}\right)$, тогда $1<\theta \leqslant \frac{3}{2}$. Используя те же аргументы, что и вьше, из неравенства Нётера-Фано вьводится

$$
n \theta\left(\Sigma_{0}+\Sigma_{1}\right)>2 n \Sigma_{0}+n \Sigma_{1}+m \Sigma_{0}^{\prime}
$$


откуда

$$
\frac{\Sigma_{1}}{\Sigma_{0}^{\prime}}>\frac{2-\theta}{\theta-1} \frac{\Sigma_{0}}{\Sigma_{0}^{\prime}}+\frac{1}{\theta-1} \frac{m}{n}
$$

так что

$$
\psi(\Gamma)>\frac{(2-\theta)^{2}}{\theta-1}\left(\frac{\Sigma_{0}}{\Sigma_{0}^{\prime}}+\frac{m}{n}\right) \geqslant x \psi(\theta),
$$

где $\psi(\theta)=(2-\theta)^{2} /(\theta-1)$. Таким образом, получаем еще одну оценку

$$
\alpha>\frac{2 n^{2}((1+\psi(\theta) / 2) x-1)}{1-(1-y) x} .
$$

Предположим, что $\theta \leqslant \frac{69}{50}$. Тогда $\psi(\theta)>1$ и мы опять приходим к противоречию с неравенством (3.6) (при любых $x$ и $y$ "наихудший" вариант - когда $x=1$ и $\left.y=\frac{1}{2}\right): \alpha>2 n^{2}$. Значит, всегда $\theta>\frac{69}{50}$.

Теперь рассмотрим случаи $k=2$ и $k=3$ при условии, что $L>k$. Пусть сначала $k=2, L>k$. Тогда $\Sigma_{0}^{\prime}=r_{1}+r_{2}$. Положим

$$
\begin{aligned}
& r_{1}=r_{2}+\cdots+r_{l}, \\
& r_{2}=r_{3}+\cdots+r_{i},
\end{aligned}
$$

где $i, l \leqslant L$. Отсюда $\Sigma_{0}^{\prime} \leqslant 3\left(r_{3}+\cdots+r_{L}\right)$, так что

$$
\Sigma_{0}=r_{1}+\cdots+r_{L}=\Sigma_{0}^{\prime}+r_{3}+\cdots+r_{L} \geqslant \frac{4}{3} \Sigma_{0}^{\prime}
$$

Таким образом, $x \geqslant \frac{4}{3}$; используя тот факт, что $\theta \leqslant \frac{3}{2}$, откуда $\psi(\theta) \geqslant \frac{1}{2}$, и неравенство (3.6), получаем противоречие ( $y$ можно полагать равньгм $\frac{1}{2}$ ):

$$
2 n^{2} \geqslant \alpha>\frac{2 n^{2}((1+1 / 4) \cdot 4 / 3-1)}{1-1 / 2 \cdot 4 / 3}=4 n^{2} .
$$

Пусть теперь $k=3, L>k$. Тогда $\Sigma_{0}^{\prime}=r_{1}+r_{2}+r_{3}$ и, как и выше, полагая

$$
\begin{aligned}
& r_{1}=r_{2}+\cdots+r_{l}, \\
& r_{2}=r_{3}+\cdots+r_{i}, \\
& r_{3}=r_{4}+\cdots+r_{j}
\end{aligned}
$$

для некоторых $i, j, l \leqslant L$, получаем $\Sigma_{0}^{\prime} \leqslant 7\left(r_{4}+\cdots+r_{L}\right)$, откуда $\Sigma_{0} \geqslant \frac{8}{7} \Sigma_{0}^{\prime}$, т.е. $x \geqslant \frac{8}{7}$, и снова приходим к противоречию ввиду оценки (3.6): $2 n^{2} \geqslant \alpha>2 n^{2}$. 


\section{§4. Трудные случаи}

Подведем предварительные итоги. Доказывая теорему 2.3 “от противного”, мы предположили, что $n>n^{\prime}$. Отсюда вывели, что линейная система $\mathscr{D}$ имеет бесконечно близкую особенность, удовлетворяющую уточненному неравенству Нётера-Фано (3.2). Далее, было показано, что через точку $B_{0}$ обязательно проходит сечение класса $s_{0}$, центр второго раздутия $B_{1} \subset V^{1}$ "задевает" собственный прообраз слоя $F^{1}$ (т.е. $\left.B_{1} \cap F^{1} \neq \varnothing\right)$. При этом "усредненная кратность" $\theta=\left(\nu_{1}+\nu_{2}\right) /(2 n)$ удовлетворяет условию $\theta \leqslant \frac{3}{2}$, откуда, учитывая, что $\nu_{1} \geqslant \nu_{2}$, получаем $\nu_{2} \leqslant \frac{3}{2} n$.

Оставшиеся случаи разбиваются на два класса: $k=L$ и $L>k \geqslant 4$. При этом если $k \geqslant 2$, то и в той, и в другой ситуации $\theta>\frac{69}{50}$ и, кроме того, $\nu_{L+1}>\frac{13}{10} n$.

Теперь выделим еще 2 ситуации. В первой точка $B_{0}$ не лежит на дивизоре ветвления $R_{V} \subset V$. Легко видеть, что это возможно только для многообразий типа $\mathscr{M}_{3}$. Действительно, многообразия $\mathscr{M}_{1}$ и $\mathscr{M}_{2}$ содержат единственную кривую класса $s_{0}$, которая необходимым образом лежит на $R_{V}$. На $\mathscr{M}_{3}$ указанное условие означает, что $B_{0} \in G_{V} \backslash R_{V}$, т.е. $B_{0}$ вне четырех сечений класса $s_{0}$, лежащих на $R_{V}$. Во второй ситуации $B_{0} \in R_{V}$.

Ключевой прием, который будет использоваться, заключается в следующем. Мы рассматриваем кривую $\left.D^{i}\right|_{F^{i}}$, являющуюся ограничением общего элемента собственного прообраза $\mathscr{D}^{i}$ линейной системы $\mathscr{D}$ на $i$-м этаже на собственный прообраз $F^{i}$ слоя, содержашего максимальную особенность. Тогда в некоторой точке $\bar{B}_{i} \in F^{i}$ кривая $\left.D^{i}\right|_{F^{i}}$ будет иметь кратность $\bar{\nu}_{i+1} \geqslant \nu_{i+1}$, что даст возможность эффективно оценить $\nu_{i+1}$. Точки $\bar{B}_{i}$ выбираются следующим образом. Если $B_{i} \in F^{i}$ - точка, то $\bar{B}_{i}=B_{i}$; если $B_{i}$ - кривая, то либо $B_{i} \not \subset F^{i}$ и тогда $\bar{B}_{i}=B_{i} \cap F^{i}$ (пересечением $B_{i}$ и $F^{i}$ будет точка по предложению 1.1, п. 2)), либо $B_{i} \subset F^{i}$ и тогда в качестве $\bar{B}_{i}$ выбирается любая точка $B_{i}$ (на самом деле, последний случай никогда не реализуется, как это будет видно из дальнейших рассмотрений). Заметим, что точка $\bar{B}_{0}$ обязательно неособая, таким образом, и остальные $\bar{B}_{i}$ неособы.

Ограничивая собственные прообразы линейной системы на собственные прообразы слоя, мы будем пользоваться следующей леммой.

Лемма 4.1. Пусть $X$-росток неособого трехмерного многообразия, $S \subset X$ - неособая поверхность и $D$ - дивизор на $X, S \not \subset \operatorname{Supp} D$.

1) Если $\sigma: X^{\prime} \rightarrow X$ - раздутие точки $B \in S \cap D, E \subset X^{\prime}$ - его исключительный дивизор, $D^{\prime}$ и $S^{\prime}$ - собственные прообразы, $е=S^{\prime} \cap E-$ соответствующая (-1)-кривая, то

$$
\left.D^{\prime}\right|_{F^{\prime}}=\left(\left.\sigma\right|_{S^{\prime}}\right)_{*}^{-1}\left(\left.D\right|_{F}\right)+m e
$$

әде $m$ - неотрицательное челое число, удовлетворяющее соотношению

$$
\left.\operatorname{mult}_{B} D\right|_{F}=\operatorname{mult}_{B} D+m .
$$

2) Если $\sigma: X^{\prime} \rightarrow X-$ - аздутие неособой кривой $B \subset D$, трансверсальной $к S$ в точке $\bar{B}, E \subset X^{\prime}$ - исключительный дивизор, $D^{\prime}$ и $S^{\prime}$ - 
собственнье прообразы, $е=S^{\prime} \cap E-$ соответствующая (-1)-кривая, mo

$$
\left.D^{\prime}\right|_{F^{\prime}}=\left(\left.\sigma\right|_{S^{\prime}}\right)_{*}^{-1}\left(\left.D\right|_{F}\right)+m e
$$

причем т удовлетворяет соотношению

$$
\left.\operatorname{mult}_{B} D\right|_{F}=\operatorname{mult}_{B} D+m \text {. }
$$

ДокАЗАТЕльство элементарно: в первом случае все сводится к очевидной двумерной ситуации ограничением $D$ и $S$ на росток общей поверхности, проходящей через точку $B$ (факт верен и в более обшей ситуации [4; лемма 6.1]); во втором достаточно записать в локальных координатах уравнения для $B, D$ и $S$ и применить формулы для раздутия кривой $B$. Лемма доказана.

Далее, рассмотрим кривую $l \in\left|-K_{F}-\bar{B}_{0}-\bar{B}_{1}\right|$. Другими словами, $l \subset F-$ антиканоническая кривая на $F$, проходящая через точку $B_{0}$ и такая, что $\bar{B}_{1} \in l^{1}$. Из условий $\mathscr{C} \mathscr{M}_{1}, \mathscr{C} \mathscr{M}_{2}$ и $\mathscr{C} \mathscr{M}_{3}$ следует, что

1) если $B_{0} \in R_{V}$, то $l$

a) либо рациональная кривая с каспидальной особенностью,

b) либо рациональная кривая с нодальной особенностью,

c) либо пара неособых рациональных кривых, пересекающихся в двух различных неособъц точках $F$,

d) либо пара неособых рациональных кривых, пересекающихся в двух различныл точках $F$, одна из которых особая,

е) либо неособая кривая рода 1 ;

2) если $B_{0} \notin R_{V}$, то $l$

f) либо пара неособых рациональных кривых, вдоль которых $F$ неособо,

g) либо пара неособых рациональных кривых, проходящих через особую точку слоя,

h) либо неприводимая кривая.

Разберем каждый из случаев, предполагая сначала, что $L>k \geqslant 4$. Ситуация $L=k$ будет рассмотрена в следующем параграфе.

a) $l$ - рациональная кривая c “каспой". Рассмотрим общий элемент $D \in \mathscr{D}$. Очевидно, $\left.D\right|_{F} \sim n\left(-K_{F}\right)$ и мы можем полагать

$$
\left.D\right|_{F}=\mu l+C
$$

где $0 \leqslant \mu \leqslant n$ и кривая $C \sim(n-\mu)\left(-K_{F}\right)$ удовлетворяет условию $l \not \subset \operatorname{Supp} C$. Положим $\alpha_{i}=\operatorname{mult}_{\bar{B}_{i}} C^{i} ;$ в частности, $\alpha_{0}=\operatorname{mult}_{\bar{B}_{0}} C$. Очевидно, $\alpha_{0} \geqslant \alpha_{1} \geqslant$ $\alpha_{2} \geqslant \cdots$. Кроме того, пусть $e_{i}=\left.E_{i}\right|_{F^{i}}$. Посмотрим теперь, что происходит на нижних этажах цепочки раздутий. Взаимное расположение кривых на этажах с "нулевого" по “третий” показано на рис. 1. 

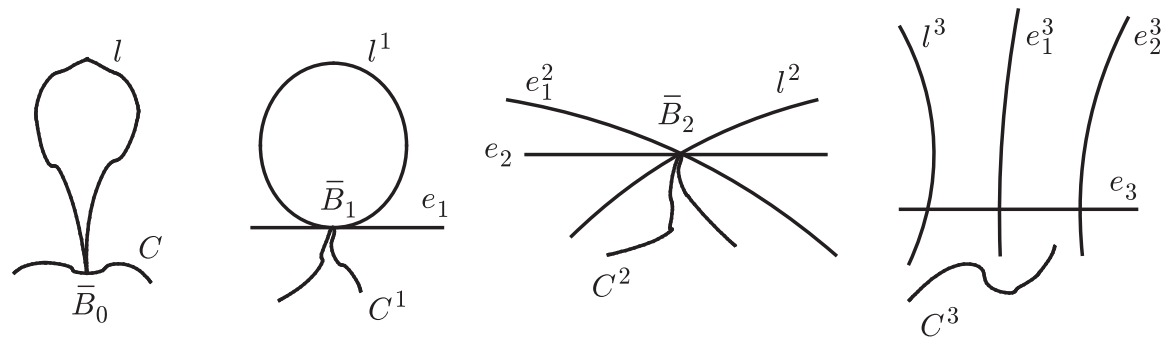

Рис. 1.

Таким образом, мы имеем

$$
\begin{aligned}
\left.D\right|_{F} & =\mu l+C \\
\left.D^{1}\right|_{F^{1}} & =\mu l^{1}+C^{1}+m_{1} e_{1}, \\
\left.D^{2}\right|_{F^{2}} & =\mu l^{2}+C^{2}+m_{1} e_{1}^{2}+m_{2} e_{2}, \\
\left.D^{3}\right|_{F^{3}} & =\mu l^{3}+C^{3}+m_{1} e_{1}^{3}+m_{2} e_{2}^{3}+m_{3} e_{3} .
\end{aligned}
$$

Используя лемму 4.1, получаем следуюшее. На нулевом этаже (на исходном многообразии)

$$
2 n \geqslant \bar{\nu}_{1}=\nu_{1}+m_{1}=2 \mu+\alpha_{0},
$$

причем $2(n-\mu)=l \circ C \geqslant 2 \alpha_{0}$, т.е. $\alpha_{0} \leqslant n-\mu$, и так как $\theta>\frac{69}{50}$ и $\nu_{1} \geqslant \theta n$, имеем $m_{1}<\frac{31}{50} n$.

На первом этаже

$$
\nu_{2}+m_{2}=\nu_{2}=\mu+\alpha_{1}+m_{1}
$$

причем $\alpha_{1} \leqslant \alpha_{0} \leqslant(n-\mu)$, откуда

$$
\frac{69}{25} n+m_{1}+m_{2}<\bar{\nu}_{1}+\bar{\nu}_{2}=3 \mu+\alpha_{0}+\alpha_{1}+m_{1} \leqslant 2 n+\mu+m_{1}
$$

Таким образом, $\frac{19}{25} n+m_{2}<\mu$, отсюда $\mu>\frac{19}{25} n$ и $m_{2}<n-\frac{19}{25} n=\frac{6}{25} n$.

Рассмотрим второй этаж. Предположим сначала, что $\bar{B}_{2} \notin l^{2}$ (или, эквивалентно, $\bar{B}_{2} \notin e_{1}^{2}$, поскольку $\bar{B}_{2} \in e_{2}$ всегда). Тогда

$$
\bar{\nu}_{3}=m_{2}+\alpha_{2} \leqslant m_{2}+\alpha_{0} \leqslant m_{2}+n-\mu<\frac{6}{25} n+\frac{6}{25} n<n
$$

получаем противоречие, поэтому всегда $\bar{B}_{2}=l^{2} \cap e_{1}^{2} \cap e_{2}$. Таким образом, $\nu_{3}+m_{3}=\bar{\nu}_{3}=\mu+\alpha_{2}+m_{1}+m_{2}$, откуда

$$
\begin{aligned}
& \frac{69}{25} n+m_{1}+m_{2}+\bar{\nu}_{3}<2 \theta n+m_{1}+m_{2}=\bar{\nu}_{1}+\bar{\nu}_{2}+\bar{\nu}_{3} \\
& \quad=4 \mu+\alpha_{0}+\alpha_{1}+\alpha_{2}+2 m_{1}+m_{2} \leqslant 4 \mu+3(n-\mu)+2 m_{1}+m_{2} \\
& \quad \leqslant 4 n+2 m_{1}+m_{2},
\end{aligned}
$$


т.е. $\bar{\nu}_{3}<4 n-\frac{69}{25} n+m_{1}<\frac{31}{25} n+\frac{31}{50} n=\frac{93}{50} n$, и так как $\nu_{3}>\frac{13}{10} n$, то $m_{3}<\frac{14}{25} n$. Кроме того, $2 \alpha_{0}+\alpha_{1}+\alpha_{2} \leqslant l \circ C=2(n-\mu)$, так что $\alpha_{2} \leqslant \frac{1}{2}(n-\mu)<\frac{3}{25} n$.

Переместимся на третий этаж. Покажем сначала, что $\bar{B}_{3} \in l^{3}$. Предположим противное, тогда, учитывая, что кривые $l^{3}, e_{1}^{3}$ и $e_{2}^{3}$ не пересекаются, имеем следующие варианты.

Пусть $\bar{B}_{3} \in e_{1}^{3}$. В этом случае

$$
\bar{\nu}_{4}=m_{1}+\alpha_{3}+m_{3} \leqslant m_{1}+\alpha_{2}+m_{3} \leqslant \frac{31}{50} n+\frac{14}{25} n+\frac{3}{25} n=\frac{13}{10} n,
$$

что противоречит условию $\bar{\nu}_{4} \geqslant \nu_{4}>\frac{13}{10} n$.

В случае $\bar{B}_{3} \in e_{2}^{3}$ также получаем противоречие:

$$
\bar{\nu}_{4}=m_{2}+m_{3}+\alpha_{3}<\frac{6}{25} n+\frac{14}{25} n+\frac{3}{25} n<n .
$$

Наконец, ситуация $\bar{B}_{3} \notin l^{3} \cup e_{1}^{3} \cup e_{2}^{3}$ невозможна по тем же соображениям:

$$
\bar{\nu}_{4}=m_{3}+\alpha_{3}<n
$$

Таким образом, всегда $\bar{B}_{3}=l^{3} \cap e_{3}$ и тогда $\bar{\nu}_{4}=\mu+\alpha_{3}+m_{3}$. Предположим сначала, что $k=4$ (и $L>k$ по условию). Используя структуру графа Г и тот факт, что $\bar{B}_{2} \in e_{1}^{2}$ и $\bar{B}_{3} \notin e_{1}^{3} \cup e_{2}^{3}$, получаем

$$
\begin{aligned}
& r_{4}=r_{5}+\cdots \leqslant r_{5}+\cdots+r_{L}, \\
& r_{3}=r_{4}+\cdots \leqslant 2\left(r_{5}+\cdots+r_{L}\right), \\
& r_{2}=r_{3} \leqslant 2\left(r_{5}+\cdots+r_{L}\right), \\
& r_{1}=2 r_{3} \leqslant 4\left(r_{5}+\cdots+r_{L}\right),
\end{aligned}
$$

так что $\Sigma_{0}^{\prime} \leqslant 9\left(r_{5}+\cdots+r_{L}\right)$ и $\Sigma_{0}=\Sigma_{0}^{\prime}+r_{5}+\cdots+r_{L} \geqslant \frac{10}{9} \Sigma_{0}^{\prime}$. Кроме того, $\Sigma_{0}=r_{1}+r_{2}+r_{3}+r_{4}+\cdots+r_{L}=2 r_{1}+r_{4}+\cdots+r_{L} \geqslant 2 r_{1}+r_{3}=\frac{5}{2} r_{1}$. Противоречие достигается использованием оценки (3.6) с параметрами $x=\frac{10}{9}$, $y=\frac{2}{5}$ и $\theta=\frac{3}{2}$ :

$$
\alpha>\frac{2 n^{2}((1+\psi(3 / 2) / 2) 10 / 9-1)}{1-3 / 5 \cdot 10 / 9}=\frac{2 n^{2}(25 / 18-1)}{1 / 3}>2 n^{2} .
$$

Таким образом, $L>k>4$. Заметим, что $\bar{\nu}_{4}=\nu_{4}+m_{4}=\mu+\alpha_{3}+m_{3}<n+m_{3}$ и $\nu_{4}>\frac{13}{10} n$, откуда $m_{4}<\frac{13}{50} n$. Теперь, поскольку $\bar{B}_{3}=l^{3} \cap e_{3}$, точка $\bar{B}_{4} \in F^{4}$ лежит либо на кривой $l^{4}$, либо на кривой $e_{3}^{4}$, либо ни на той, ни на другой. Итак, если $\bar{B}_{4} \in l^{4}$, то $\bar{\nu}_{5}=\mu+\alpha_{4}+m_{4}<n+\frac{13}{50} n=\frac{63}{50} n$, что невозможно, так как $\bar{\nu}_{5} \geqslant \nu_{5}>\frac{13}{10} n$. Если $\bar{B}_{4} \in e_{3}^{4}$, мы также немедленно приходим к противоречию:

$$
\bar{\nu}_{5}=\alpha_{4}+m_{4}+m_{3}<\frac{3}{25} n+\frac{13}{50} n+\frac{14}{25} n=\frac{47}{50} n<n .
$$



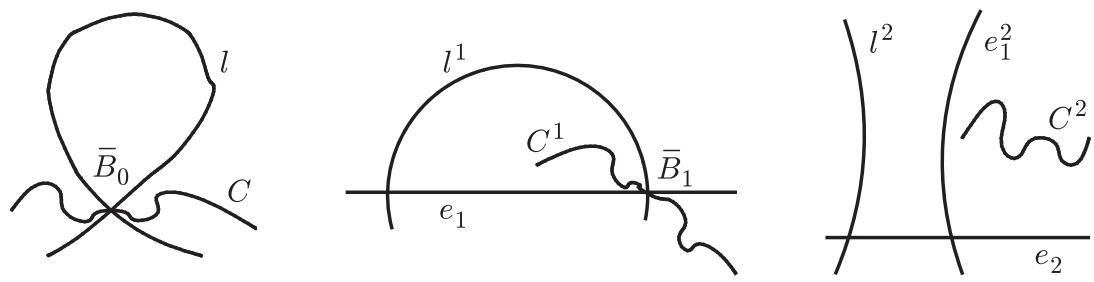

Рис. 2.

Наконец, если $\bar{B}_{4} \notin l^{4} \cup e_{3}^{4}$, то $\bar{\nu}_{5}=\alpha_{4}+m_{4}<n$.

Итак, $l$ не может быть рациональной кривой с каспидальной особенностью в точке $\bar{B}_{0}$.

ЗАмЕчАнИЕ 4.2. Из рассмотрения кратностей на нулевом и первом этажах следует, что $\bar{\nu}_{1}+\bar{\nu}_{2}=3 \mu+\alpha_{0}+\alpha_{1}+m_{1} \leqslant 2 n+\mu+m_{1} \leqslant 3 n+m_{1}$, откуда $\nu_{1}+\nu_{2} \leqslant 3 n$. Таким образом, лемма 3.5 доказана в ситуации а).

b) $l$ - рациональная кривая $c$ нодальной особенностью. Рассуждения близки к предыдущим. Мы также придерживаемся тех же обозначений. Геометрическая ситуация на нижних этажах воспроизведена на рис. 2.

Итак,

$$
\begin{aligned}
\left.D\right|_{F} & =\mu l+C, \\
\left.D^{1}\right|_{F^{1}} & =\mu l^{1}+C^{1}+m_{1} e_{1}, \\
\left.D^{2}\right|_{F^{2}} & =\mu l^{2}+C^{2}+m_{1} e_{1}^{2}+m_{2} e_{2},
\end{aligned}
$$

где $C \sim(n-\mu)\left(-K_{F}\right)$ и $l \not \subset \operatorname{Supp} C$.

На нулевомэтаже, очевидно, $\bar{\nu}_{1}=2 \mu+\alpha_{0}$. Далее, $\alpha_{0} \leqslant n-\mu$, так что $\mu+\alpha_{i} \leqslant n$ для всех $i$. Поскольку $\bar{\nu}_{1} \leqslant 2 n$ и $\nu_{1}>\frac{69}{50} n$, то $m_{1}<\frac{31}{50} n$.

На первом этаже $\bar{\nu}_{2}=\mu+\alpha_{1}+m_{1}$. Так как $\alpha_{1} \leqslant \alpha_{0}$ и $\theta>\frac{69}{50}$, то

$$
\frac{69}{25} n+m_{1}+m_{2}<\bar{\nu}_{1}+\bar{\nu}_{2}=3 \mu+\alpha_{0}+\alpha_{1}+m_{1} \leqslant 2 n+\mu+m_{1},
$$

откуда $m_{2}<\frac{6}{25} n, \mu>\frac{19}{25} n$ и $\alpha_{i} \leqslant n-\mu<\frac{6}{25} n$.

На втором этаже $l^{2}$ и $e_{1}^{2}$ разведены, так что

i) если $\bar{B}_{2} \in l^{2}$, то $\frac{13}{10} n<\nu_{3} \leqslant \bar{\nu}_{3}=\mu+\alpha_{2}+m_{2} \leqslant n+\frac{6}{25} n<\frac{13}{10} n$, противоречие;

ii) если $\bar{B}_{2} \in e_{1}^{2}$, то $\frac{13}{10} n<\bar{\nu}_{3}=m_{1}+\alpha_{2}+m_{2}<\frac{31}{50} n+\frac{6}{25} n+\frac{6}{25} n<\frac{13}{10} n$, снова противоречие;

iii) если $\bar{B}_{2} \notin l^{2} \cup e_{1}^{2}$, то $\bar{\nu}_{3}=\alpha_{2}+m_{2}<n$, что также невозможно.

Итак, случай b) не реализуется.

ЗАмечание 4.3 . Те же аргументы, что и в замечании 4.2 , показывают, что $\nu_{1}+\nu_{2} \leqslant 3 n$, так что лемма 3.5 верна и для случая $\left.\mathrm{b}\right)$. 
c) $l$ - пара неособых рациональных кривых, пересекаюшихся в двух различных неособых точках $F$. Пусть $l=l_{1} \cup l_{2}$, причем $\bar{B}_{1} \in l_{1}^{1}$ (и, очевидно, $\bar{B}_{1} \notin l_{2}^{1}$ ), и

$$
\left.D\right|_{F}=\mu_{1} l_{1}+\mu_{2} l_{2}+C
$$

где $l_{1}, l_{2} \not \subset \operatorname{Supp} C$. Тогда

$$
\begin{aligned}
& \left.D^{1}\right|_{F^{1}}=\mu_{1} l_{1}^{1}+\mu_{2} l_{2}^{1}+C^{1}+m_{1} e_{1} \\
& \left.D^{2}\right|_{F^{2}}=\mu_{1} l_{1}^{2}+\mu_{2} l_{2}^{2}+C^{2}+m_{1} e_{1}^{2}+m_{2} e_{2}, \\
& \left.D^{3}\right|_{F^{3}}=\mu_{1} l_{1}^{3}+\mu_{2} l_{2}^{3}+C^{3}+m_{1} e_{1}^{3}+m_{2} e_{2}^{3}+m_{3} e_{3} .
\end{aligned}
$$

Ситуация изображена на рис. 3.

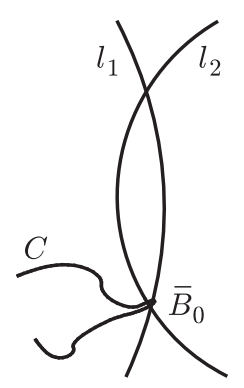

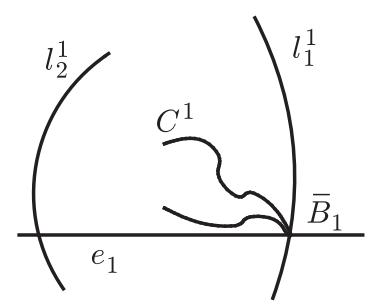

Рис. 3.

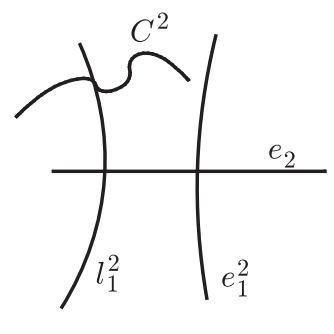

На нулевом этаже $\bar{\nu}_{1}=\mu_{1}+\mu_{2}+\alpha_{0}$. Далее, так как $l_{1}$ и $l_{2}$ являются $(-1)$-кривьги, то

$$
\begin{aligned}
& \left(\left.D\right|_{F}-\mu_{1} l_{1}-\mu_{2} l_{2}\right) \circ l_{1}=n+\mu_{1}-2 \mu_{2} \geqslant \alpha_{0}, \\
& \left(\left.D\right|_{F}-\mu_{1} l_{1}-\mu_{2} l_{2}\right) \circ l_{2}=n-2 \mu_{1}+\mu_{2} \geqslant \alpha_{0},
\end{aligned}
$$

откуда, суммируя эти два неравенства, получаем $\alpha_{0} \leqslant n-\frac{1}{2}\left(\mu_{1}+\mu_{2}\right)$, а подставляя одно в другое и заменяя $\alpha_{0}$ нулем, находим, что

$$
0 \leqslant n+\mu_{1}-2 \mu_{2} \leqslant n+\mu_{1}-4 \mu_{1}+2 n=3 n-3 \mu_{1},
$$

т.е. $\mu_{1} \leqslant n$. Аналогично показывается, что $\mu_{2} \leqslant n$. Также из предположения $\nu_{1}>\frac{69}{50} n$ и неравенства $\bar{\nu}_{1} \leqslant 2 n$ выводим, что $m_{1}<\frac{31}{50} n$.

На первом этаже $\bar{\nu}_{2}=\mu_{1}+\alpha_{1}+m_{1}$. Далее, ясно, что

$$
\left(\left.D\right|_{F}-\mu_{1} l_{1}-\mu_{2} l_{2}\right) \circ l_{1}=n+\mu_{1}-2 \mu_{2} \geqslant \alpha_{0}+\alpha_{1},
$$

откуда, учитывая $n-2 \mu_{1}+\mu_{2} \geqslant \alpha_{0}$, находим, что

$$
\alpha_{0}+\alpha_{1} \leqslant \frac{2}{3}\left(2 \alpha_{0}+\alpha_{1}\right) \leqslant \frac{4}{3} n-\frac{2}{3}\left(\mu_{1}+\mu_{2}\right)
$$


Таким образом,

$$
\begin{aligned}
\frac{69}{25} n+m_{1}+m_{2} & <\bar{\nu}_{1}+\bar{\nu}_{2}=2 \mu_{1}+\mu_{2}+\alpha_{0}+\alpha_{1}+m_{1} \leqslant \frac{4}{3} n+\frac{4}{3} \mu_{1}+\frac{1}{3} \mu_{2}+m_{2} \\
& \leqslant \frac{5}{3} n+\frac{4}{3} \mu_{1}+m_{1},
\end{aligned}
$$

откуда $m_{2}<3 n-\frac{69}{25} n=\frac{6}{25} n$ и $\mu_{1}>\frac{3}{4}\left(\frac{69}{25} n-\frac{5}{3} n\right)=\frac{41}{50} n$. Заметим также, что $\nu_{1}+$ $\nu_{2} \leqslant 3 n$, поскольку $\mu_{1} \leqslant n$. Кроме того, $\alpha_{i} \leqslant \alpha_{0} \leqslant n-2 \mu_{1}+\mu_{2} \leqslant 2 n-2 \mu_{1}<\frac{9}{25} n$.

Покажем, что $\alpha_{i}+\mu_{1} \leqslant n$ при $i \geqslant 1$. Действительно, так как $\alpha_{0}+\mu_{1} \leqslant n-$ $\mu_{1}+\mu_{2}$, то утверждение очевидно, если $\mu_{1} \geqslant \mu_{2}$. Пусть $\mu_{1}<\mu_{2}$. Раз $\alpha_{0}+\alpha_{1} \leqslant n+$ $\mu_{1}-2 \mu_{2}$, то $2 \mu_{1}+\alpha_{0}+\alpha_{1} \leqslant n+3 \mu_{1}-2 \mu_{2}<n+\mu_{1} \leqslant 2 n$, откуда $\mu_{1}+\alpha_{1}<n$.

Рассмотрим второй этаж. Заметим, что $l_{1}^{2}$ и $e_{1}^{2}$ не пересекаются. Таким образом, если $\bar{B}_{2} \in e_{1}^{2}$, то $\bar{\nu}_{3}=m_{1}+\alpha_{2}+m_{2}<\frac{31}{50} n+\frac{9}{25} n+\frac{6}{25} n=\frac{61}{50} n<\frac{13}{10} n$, что противоречит условию $\nu_{3}>\frac{13}{10} n$. Тем более невозможен случай $\bar{B}_{2} \notin l_{1}^{2} \cup e_{1}^{2}$ : тогда $\bar{\nu}_{3}=\alpha_{2}+m_{3}<n$.

Наконец, пусть $\bar{B}_{2} \in l_{1}^{2}$. Так как $\mu_{1}+\alpha_{2} \leqslant n$, то $\bar{\nu}_{3}=\mu_{1}+\alpha_{2}+m_{2}<n+$ $\frac{6}{25} n=\frac{31}{25} n$, что опять же противоречит условию $\nu_{3}>\frac{13}{10} n$.

Таким образом, ситуация с) невозможна.

ЗАмЕчАниЕ 4.4. Как и в предыдущих случаях, было показано, что $\nu_{1}+\nu_{2} \leqslant$ $3 n$, и лемма 3.5 в этой части также доказана.

d) $l$ - пара неособых рациональных кривых, пересекаюшихся в двух различных точках $F$, одна из которьх особая. Понятно, что $\bar{B}_{0}$ соответствует неособой точке пересечения. Этот случай мало чем отличается от предыдущего, и можно дословно использовать те же рассуждения, так что проверка оставляется читателю. Скажем лишь, что для начала надо разрешить особую точку $F$ (например, раздув соответствуюшую точку на $V$ ) и ограничить все на собственный прообраз слоя $\widetilde{F}$. Тогда если $\widetilde{l}_{1}, \widetilde{l}_{2}, \widetilde{D}$ - соответствуюшие собственные прообразы, а $e$-исключительный дивизор на $\widetilde{F}$ (это $(-2)$-кривая), то $\widetilde{l}_{1}$ и $\widetilde{l}_{2}$ суть $(-1)$-кривые, пересекаюшиеся в одной точке, и выполняются следующие соотношения:

$$
\begin{gathered}
\widetilde{l}_{1}=\left(l_{1}\right)^{*}-\frac{1}{2} e, \\
\widetilde{l}_{2}=\left(l_{2}\right)^{*}-\frac{1}{2} e, \\
\widetilde{D}=D^{*}-\frac{1}{2}\left(\mu_{1}+\mu_{2}+\varepsilon\right) e, \quad \varepsilon \geqslant 0, \\
\left(\widetilde{D}-\mu_{1} \widetilde{l}_{1}-\mu_{2} \widetilde{l}_{2}\right) \circ \widetilde{l}_{1}=n+\frac{1}{2} \mu_{1}-\frac{3}{2} \mu_{2} \geqslant \alpha_{0}+\alpha_{1}, \\
\left(\widetilde{D}-\mu_{1} \widetilde{l}_{1}-\mu_{2} \widetilde{l}_{2}\right) \circ \widetilde{l}_{2}=n-\frac{3}{2} \mu_{1}+\frac{1}{2} \mu_{2} \geqslant \alpha_{0},
\end{gathered}
$$

где символ “*” означает полный прообраз. Далее работает схема рассуждений предыдушего случая. В частности, получаются те же основные соотношения, а именно,

$$
\begin{gathered}
\alpha_{0}+\alpha_{1} \leqslant \frac{4}{3} n-\frac{1}{2}\left(\mu_{1}+\mu_{2}\right), \\
\alpha_{i}+\mu_{1} \leqslant n, \quad i \geqslant 1,
\end{gathered}
$$


и те же основные оценки $\mu_{1}, \mu_{2} \leqslant n, m_{1}<\frac{31}{50} n, \mu_{1}>\frac{41}{50} n, m_{2}<\frac{6}{25} n, \nu_{1}+\nu_{2} \leqslant 3 n$. Единственное отличие

$$
\alpha_{i} \leqslant \alpha_{0} \leqslant n-\frac{3}{2} \mu_{1}+\frac{1}{2} \mu_{2}<\frac{27}{100} n
$$

но эта оценка еше сильнее, чем в случае с). Таким образом, случай d) также исключается.

е) $l$-неособая кривая рода 1. Здесь совсем просто. Пусть

$$
\begin{aligned}
\left.D\right|_{F} & =\mu l+C \\
\left.D^{1}\right|_{F^{1}} & =\mu l^{1}+C^{1}+m_{1} e_{1} .
\end{aligned}
$$

Тогда

$$
\begin{aligned}
\bar{\nu}_{1} & =\mu+\alpha_{0} \\
\bar{\nu}_{2} & =\mu+\alpha_{1}+m 1 \\
\alpha_{0}+\alpha_{1} & \leqslant\left(\left.D\right|_{F}-\mu l\right) \circ l=2(n-\mu)
\end{aligned}
$$

и мы немедленно получаем противоречие:

$$
\bar{\nu}_{1}+\bar{\nu}_{2}=2 \mu+\alpha_{0}+\alpha_{1}+m_{1} \leqslant 2 n+m_{1}
$$

т.е. $\nu_{1}+\nu_{2} \leqslant 2 n$. Таким образом, случай е) исключен.

f) $l$ - пара неособых рациональных кривых, вдоль которых $F$ неособо, $B_{0} \in$ $l \backslash R_{V}$. Пусть $l=l_{1} \cup l_{2}$. Так как $B_{0} \notin R_{V}$, то $\bar{B}_{0}$ лежит только на одной из двух кривых, скажем, на $l_{1}$. Заметим, что $\left(l_{1}\right)^{2}=\left(l_{2}\right)^{2}=-1$ и $l_{1} \circ l_{2}=2$.

Итак, на нулевом этаже мы можем положить

$$
\left.D\right|_{F}=\mu_{1} l_{1}+\mu_{2} l_{2}+C
$$

где $\mu_{1}, \mu_{2} \geqslant 0$ и $l_{1}, l_{2} \not \subset \operatorname{Supp} C$. Очевидно, $\bar{\nu}_{1}=\mu_{1}+\alpha_{0}$. Кроме того,

$$
0 \leqslant\left(\left.D\right|_{F}-\mu_{1} l_{1}-\mu_{2} l_{2}\right) \circ l_{2}=n-2 \mu_{1}+\mu_{2}
$$

откуда $\mu_{1} \leqslant \frac{1}{2}\left(n+\mu_{2}\right)$.

На первом этаже

$$
\left.D^{1}\right|_{F^{1}}=\mu_{1} l_{1}^{1}+\mu_{2} l_{2}^{1}+C^{1}+m_{1} e_{1}
$$

для некоторого $m_{1} \geqslant 0$; таким образом, $\bar{\nu}_{2}=\mu_{1}+\alpha_{1}+m_{1}$. Очевидно, что

$$
\alpha_{0}+\alpha_{1} \leqslant\left(\left.D\right|_{F}-\mu_{1} l_{1}-\mu_{2} l_{2}\right) \circ l_{1}=n+\mu_{1}-2 \mu_{2}
$$

Тогда

$$
\bar{\nu}_{1}+\bar{\nu}_{2}=2 \mu_{1}+\alpha_{0}+\alpha_{1}+m_{1} \leqslant n+3 \mu_{1}-2 \mu_{2}+m_{1},
$$


откуда, учитывая неравенство для $\mu_{1}$, получаем

$$
\nu_{1}+\nu_{2} \leqslant n+\frac{3}{2}\left(n+\mu_{2}\right)-2 \mu_{2} \leqslant \frac{5}{2} n
$$

что противоречит условию $\theta>\frac{69}{50}$. Случай $\left.\mathrm{f}\right)$ исключен.

g) $l$-пара неособьхх рациональных кривьгх, проходящих через особую точку $F$, $B_{0} \in l \backslash R_{V}$. Этот случай разбирается точно так же, как и предыдущий, только сначала надо разрешить особую точку слоя. Тогда собственные прообразы $l_{1}$ и $l_{2}$ станут (-1)-кривыми, пересекающимися в одной точке, и мы получим следующие оценки:

$$
\begin{aligned}
\alpha_{0}+\alpha_{1} & \leqslant n+\frac{1}{2} \mu_{1}-\frac{3}{2} \mu_{2}, \\
0 & \leqslant n-\frac{3}{2} \mu_{1}+\frac{1}{2} \mu_{2} .
\end{aligned}
$$

Далее,

$$
\bar{\nu}_{1}+\bar{\nu}_{2}=2 \mu_{1}+\alpha_{0}+\alpha_{1}+m_{1}
$$

так что

$$
\nu_{1}+\nu_{2} \leqslant n+\frac{5}{2} \mu_{1}-\frac{3}{2} \mu_{2} \leqslant n+\frac{5}{2}\left(\frac{2}{3} n+\frac{1}{3} \mu_{2}\right)-\frac{3}{2} \mu_{2} \leqslant \frac{8}{3} n
$$

Полученное противоречит условию $\theta>\frac{69}{50}$. Случай g) невозможен.

h) $l$ - неприводимая кривая, $B_{0} \in l \backslash R_{V}$. Этот случай также не реализуется. Для доказательства можно дословно воспользоваться рассуждениями случая е).

Таким образом, все ситуации, когда $L>k$, разобраны: доказана их нереализумость. Заметим, что одновременно была доказана лемма 2.3.

\section{§5. Завершение доказательства теоремы 2.3}

Все, что нам осталось сделать, это показать, что случай $L=k$ также невозможен.

Заметим, что всегда $\theta \leqslant \frac{3}{2} n$, т.е. $\nu_{1}+\nu_{2} \leqslant 3 n$, поэтому из неравенства Нётера-Фано следует, что $\Sigma_{1}>\Sigma_{0} \geqslant L$.

Случай $L=k=1$. Здесь мы имеем $r_{1}=r_{2}=\cdots=r_{N}=1$ и $\Sigma_{1}>1$, т.е. цепочка раздутий содержит по меньшей мере два раздутия кривых. Этим самым сразу же исключаются ситуации е) и h) из предыдущего параграф̆а, а также ситуация а), когда $l$ - кривая с каспой и $B_{0} \in R_{V}$. Действительно, из вычислений для этого случая следовало, что обязательно $\bar{B}_{2}=l^{2} \cap e_{1}^{2}$. Но это противоречит п. 4) предложения 1.1: $B_{2} \cap E_{1}^{2}=\varnothing$.

Чтобы разобраться с остальными возможностями, используем формулу (3.6) в следуюшем варианте. Во-первых, положим $x=y=1$, это как раз соответствует случаю $L=k=1$. Во-вторых, вместо $\theta$ введем "расширенные" усредненные кратности. Именно, пусть

$$
\theta_{i}=\frac{1}{i n}\left(\nu_{1}+\cdots+\nu_{i}\right)
$$


В частности, $\theta=\theta_{2}$. Тогда легко видеть, что верна оценка

$$
\alpha>n^{2} \psi\left(\theta_{i}\right)
$$

при любом $i \leqslant N$, где, напомним,

$$
\psi\left(\theta_{i}\right)=\frac{\left(2-\theta_{i}\right)^{2}}{\theta_{i}-1}
$$

Таким образом, мы можем полагать, что для любого $i \leqslant N$ выполнено $\theta_{i}>\frac{5}{4}$ (иначе $\alpha>2 n^{2}$, что невозможно). Заметим, что тем самым мы немедленно избавляемся от случая f): там $\nu_{1}+\nu_{2} \leqslant \frac{5}{2} n$, т.е. $\theta_{2} \leqslant \frac{5}{4}$. Рассмотрим оставшиеся ситуации. Мы будем пользоваться обозначениями и вычислениями предыдущего параграфа, относящимся к соответствующим случаям. Читатель может также использовать данные там рисунки.

Ситуация b). Так как $2 \alpha_{0}+\alpha_{1} \leqslant 2(n-\mu)$ и $\alpha_{1} \leqslant \alpha_{0}$, то, очевидно, имеем $\alpha_{0}+\alpha_{1} \leqslant \frac{4}{3}(n-\mu)$. Тогда

$$
\frac{5}{2} n+m_{1}+m_{2}<\bar{\nu}_{1}+\bar{\nu}_{2}=3 \mu+\alpha_{0}+\alpha_{1}+m_{1} \leqslant \frac{4}{3} n+\frac{5}{3} \mu+m_{1},
$$

откуда $m_{2}<\frac{1}{2} n$ и $\mu>\frac{7}{10} n$. Поднимемся на второй этаж (так как $\Sigma_{1} \geqslant 2$, то $N$ - количество этажей в цепочке раздутий - не менњше трех). В силу п. 4) предложения $1.1 B_{2} \notin e_{1}^{2}$. Предположим сначала, что $B_{2} \notin l^{2}$. Поскольку

$$
\alpha_{2} \leqslant \frac{1}{2}\left(\alpha_{0}+\alpha_{1}\right) \leqslant \frac{2}{3}(n-\mu)<\frac{1}{5} n
$$

мы легко видим, что

$$
\bar{\nu}_{3}=\alpha_{2}+m_{2}<\frac{1}{5} n+\frac{1}{2} n<n,
$$

что невозможно. Значит, обязательно $B_{2} \in l^{2}$. Тогда

$$
2 \alpha_{0}+\alpha_{1}+\alpha_{2} \leqslant\left(\left.D\right|_{F}-\mu l\right) \circ l=2(n-\mu)
$$

откуда $\alpha_{0}+\alpha_{1}+\alpha_{2} \leqslant \frac{3}{2} n(n-\mu)$. Таким образом,

$$
\begin{aligned}
& \frac{15}{4} n+m_{1}+m_{2}+m_{3}<\bar{\nu}_{1}+\bar{\nu}_{2}+\bar{\nu}_{3} \\
& \quad=4 \mu+\alpha_{0}+\alpha_{1}+\alpha_{2}+m_{1}+m_{2} \leqslant \frac{3}{2} n+\frac{5}{2} \mu+m_{1}+m_{2}
\end{aligned}
$$

и, значит, $m_{3}<\frac{1}{4} n$ и $\mu>\frac{9}{10} n$. Кроме того, $\nu_{1}+\nu_{2}+\nu_{3} \leqslant 4 n$, т.е. $\theta_{3} \leqslant \frac{4}{3}$, а тогда из неравенства Нётера-Фано выводим, что $\Sigma_{1}>2 \Sigma_{0}=2$, т.е. $N>3$. Цепочка раздутий не обрьвается на втором этаже, и, следовательно, мы можем подняться на третий этаж. 
Пусть $B_{3} \in e_{2}^{3}$. Так как $\alpha_{3} \leqslant \alpha_{2}<\frac{1}{5} n$, то

$$
\bar{\nu}_{4}=\alpha_{3}+m_{2}+m_{3}<\frac{1}{5} n+\frac{1}{2} n+\frac{1}{4} n<n,
$$

что,очевидно, невозможно. По тем же соображениям не реализуется случай, когда $B_{3} \notin l^{3} \cup e_{2}^{3}$. Единственная оставшаяся возможность $B_{3} \in l^{3}$. Мы можем воспользоваться грубой оценкой

$$
\alpha_{0}+\alpha_{1}+\alpha_{2}+\alpha_{3} \leqslant 2(n-\mu)
$$

и тогда

$\bar{\nu}_{+} \bar{\nu}_{2}+\bar{\nu}_{3}+\bar{\nu}_{4}=5 \mu+\alpha_{0}+\alpha_{1}+\alpha_{2}+\alpha_{3}+m_{1}+m_{2}+m_{3} \leqslant 5 n+m_{1}+m_{2}+m_{3}$,

т.е. $\nu_{1}+\nu_{2}+\nu_{3}+\nu_{4} \leqslant 5 n$, откуда получаем противоречие: $\theta_{4} \leqslant \frac{5}{4}$. Случай b) разобран.

Ситуация с). Как и в предыдущем параграфе, полагаем, что $B_{1} \in l_{1}^{1}$. Так как $\alpha_{0} \leqslant n-2 \mu_{1}+\mu_{2}$ и $\alpha_{0}+\alpha_{1} \leqslant n+\mu_{1}-2 \mu_{2}$, то $2 \alpha_{0}+\alpha_{1} \leqslant 2 n-\left(\mu_{1}+\mu_{2}\right)$, откуда получаем следующие оценки:

$$
\alpha_{0}+\alpha_{1} \leqslant \frac{4}{3} n+\frac{2}{3}\left(\mu_{1}+\mu_{2}\right)
$$

и, учитьвая, что $\alpha_{i} \leqslant \alpha_{0}$,

$$
\alpha_{0}+\alpha_{1}+\alpha_{2} \leqslant 2 n-\left(\mu_{1}+\mu_{2}\right)
$$

Далее, так как $\theta_{2}>\frac{5}{4}$ и $\mu_{i} \leqslant n$, то

$$
\frac{5}{2} n+m_{1}+m_{2}<\bar{\nu}_{1}+\bar{\nu}_{2}=2 \mu_{1}+\mu_{2}+\alpha_{0}+\alpha_{1} \leqslant \frac{5}{3} n+\frac{4}{3} \mu_{1}+m_{1}
$$

поэтому $m_{2}<\frac{1}{2} n$.

Предположим, что $B_{2} \notin l^{2}$ (заметим, что $B_{2} \notin e_{1}^{2}$ по предложению 1.1). Тогда $\bar{\nu}_{1}+\bar{\nu}_{2}+\bar{\nu}_{3}=2 \mu_{1}+\mu_{2}+\alpha_{0}+\alpha_{1}+\alpha_{2}+m_{1}+m_{2} \leqslant 2 n+\mu_{1}+m_{1}+m_{2} \leqslant 3 n+m_{1}+m_{2}$, что приводит к противоречию: $\nu_{1}+\nu_{2}+\nu_{3} \leqslant 3 n$. Таким образом, $B_{2} \in l_{1}^{2}$ и, поскольку $\theta_{3}>\frac{5}{4}$, мы имеем

$$
\begin{aligned}
\frac{15}{4} n+m_{1}+m_{2}+m_{3} & <\bar{\nu}_{1}+\bar{\nu}_{2}+\bar{\nu}_{3}=3 \mu_{1}+\mu_{2}+\alpha_{0}+\alpha_{1}+\alpha_{2}+m_{1}+m_{2} \\
& \leqslant 2 n+2 \mu_{1}+m_{1}+m_{2} .
\end{aligned}
$$

Отсюда $m_{3}<\frac{1}{4} n, \mu_{1}>\frac{7}{8} n$ и тогда $\alpha_{i} \leqslant n-2 \mu_{1}+\mu_{2} \leqslant 2 n-2 \mu_{1}<\frac{1}{4} n$. Кроме того, $\nu_{1}+\nu_{2}+\nu_{3} \leqslant 4 n$, т.е. $\theta_{3}>\frac{4}{3}$, откуда $\Sigma_{1}>2 \Sigma_{0}=2$, т.е. $N>3$, и мы можем продолжить рассмотрение на третьем этаже. 
Предположим, что $B_{3} \in e_{2}^{3}$. Получаем противоречие:

$$
\bar{\nu}_{4}=\alpha_{3}+m_{2}+m_{3}<\frac{1}{4} n+\frac{1}{2} n+\frac{1}{4} n=n .
$$

Тем более невозможен случай $B_{3} \notin l_{1}^{3} \cup e_{2}^{3}$. Пусть $B_{3} \in l_{1}^{3}$. По аналогии с тем, как было сделано вьше, мы можем считать, что $\alpha_{0}+\alpha_{1}+\alpha_{2}+\alpha_{3} \leqslant 2 n-\left(\mu_{1}+\mu_{2}\right)$. Тогда

$\bar{\nu}_{1}+\cdots+\bar{\nu}_{4}=4 \mu_{1}+\mu_{2}+\alpha_{0}+\cdots+\alpha_{3}+m_{1}+m_{2}+m_{3} \leqslant 5 n+m_{1}+m_{2}+m_{3}$,

т.е. $\nu_{1}+\cdots+\nu_{4} \leqslant 5 n$, что противоречит условию $\theta_{4}>\frac{5}{4}$. Случай с) также разобран.

Ситуация d) исключается дословно теми же рассуждениями (и при помоши тех же оценок), что и с). Нам осталось рассмотреть последнюю ситуацию.

Ситуация g). Мы полагаем, что $\bar{B}_{0} \in l_{1}$. Имеем

$$
\begin{gathered}
\alpha_{0}+\alpha_{1} \leqslant n+\frac{1}{2} \mu_{1}-\frac{3}{2} \mu_{2}, \\
0 \leqslant n-\frac{3}{2} \mu_{1}+\frac{1}{2} \mu_{2},
\end{gathered}
$$

так что

$$
\begin{aligned}
\alpha_{i} \leqslant \frac{1}{2}\left(\alpha_{0}+\alpha_{1}\right) & \leqslant n-\frac{1}{2}\left(\mu_{1}+\mu_{2}\right), \quad i \geqslant 1, \\
\mu_{1} & \leqslant \frac{2}{3} n+\frac{1}{3} \mu_{2} .
\end{aligned}
$$

Так как $\theta_{2}>\frac{5}{4}$, то

$$
\begin{aligned}
\frac{5}{2} n+m_{1}+m_{2} & \leqslant \bar{\nu}_{1}+\bar{\nu}_{2}=2 \mu_{1}+\alpha_{0}+\alpha_{1}+m_{1} \leqslant n+\frac{5}{2} \mu_{1}-\frac{3}{2} \mu_{2}+m_{2} \\
& \leqslant n+\frac{5}{2}\left(\frac{2}{3} n+\frac{1}{3} \mu_{2}\right)-\frac{3}{2} \mu_{2}+m_{1} \leqslant \frac{8}{3} n+m_{1}
\end{aligned}
$$

откуда $m_{2}<\frac{1}{6} n$ и $\mu_{1}>\frac{2}{5}\left(\frac{5}{2} n-n\right)=\frac{3}{5} n$. Последняя оценка влечет $\alpha_{i} \leqslant n-$ $\frac{1}{2} \mu_{1}<\frac{7}{10} n$ при $i>0$.

Рассмотрим второй этаж. Если $B_{2} \notin l_{1}^{2}$, то $\bar{\nu}_{3}=\alpha_{2}+m_{2}<\frac{7}{10} n+\frac{1}{6} n<n$, что невозможно. Пусть $B_{2} \in l_{1}^{2}$, тогда $\alpha_{0}+\alpha_{1}+\alpha_{2} \leqslant n+\frac{1}{2} \mu_{1}-\frac{3}{2} \mu_{2}$ и, используя неравенство $\mu_{1} \leqslant \frac{2}{3} n+\frac{1}{3} \mu_{2}$, мы получаем

$$
\begin{aligned}
\bar{\nu}_{1}+\bar{\nu}_{2}+\bar{\nu}_{3} & =3 \mu_{1}+\alpha_{0}+\alpha_{1}+\alpha_{2}+m_{1}+m_{2} \\
& \leqslant n+\frac{7}{2} \mu_{1}-\frac{3}{2} \mu_{2}+m_{1}+m_{2} \leqslant \frac{10}{3} n+m_{1}+m_{2},
\end{aligned}
$$

т.е. $\nu_{1}+\nu_{2}+\nu_{3} \leqslant \frac{10}{3} n$, что противоречит условию $\theta_{3}>\frac{5}{4}$. Ситуация g), а вместе с ней и весь случай $L=k=1$ разобраны. 
Случай $L=k \geqslant 2$. Заметим, что $r_{1} \leqslant \frac{1}{2} \Sigma_{0}$ в этом случае, поскольку $L \geqslant 2$. Это значит, что мы можем использовать оценки $\theta>\frac{69}{50}$ и $\nu_{L+1}>\frac{13}{10} n$, полученные в $\S 3$. В частности, $\nu_{3}>\frac{13}{10} n$. Этим самым исключаются случаи b) $\left.-\mathrm{h}\right)$. Действительно, $\Sigma_{1}>L \geqslant 2$, т.е. мы можем подняться по цепочке раздутий, по меньшей мере, до пятого этажа. Но в перечисленных случаях мы получаем противоречие с указанньми оценками уже на третьем (или даже более низком) этаже.

Остается разобраться лишш со случаем а). Заметим, что если $L=k \geqslant 4$, то $\nu_{5} \geqslant \nu_{L+1}>\frac{13}{10} n$, а в соответствующем разделе предыдушего параграфа рассмотрение выше пятого этажа при таком условии провести не удалось: достигалось противоречие. Значит, мы можем полагать $L=k=2$ или $L=k=3$.

Отметим, что $r_{1}=r_{2}=\cdots=r_{N}=1$ при $L=k=2$, а при $L=k=3$ имеем $r_{1}=r_{2}+r_{3}\left(\right.$ так как $\left.\bar{B}_{2} \in e_{1}^{2}\right)$ и $r_{2}=r_{3}=\cdots=r_{N}=1$. Таким образом, $N \geqslant 5$ в первом случае и $N \geqslant 9$ во втором. Введем усредненные кратности

$$
\theta_{j}=\frac{1}{j n}\left(\nu_{1}+\cdots+\nu_{j}\right)
$$

Тогда $n \theta_{j}\left(\Sigma_{0}+\Sigma_{1}\right) \geqslant \sum_{i=1}^{N} r_{i} \nu_{i}$ при всех $j \leqslant N$ в первом случае и $j \leqslant 5$ во втором (для доказательства последнего достаточно использовать тот факт, что $r_{1}=r_{2}+r_{3}$ и $\left.N \geqslant 9\right)$. Таким образом, вывод оценки (3.6) из (3.5) остается верен при замене $\theta$ на $\theta_{j}$ для $j \leqslant 5$, так что из (3.6) при $x=1$ и $y=\frac{1}{2}$ мы имеем

$$
\alpha>2 n^{2} \psi\left(\theta_{j}\right)
$$

и, следовательно, можем дополнительно полагать, что $\theta_{j}>\frac{69}{50}$.

Рассмотрим сначала случай $L=k=3$. Так как $\nu_{4}>\frac{13}{10} n$ по условию, все рассуждения из соответствующего места $\S 4$ остаются верными вплоть до третьего этажа. На четвертом этаже возможности $\bar{B}_{4} \in e_{3}^{4}$ и $\bar{B}_{4} \notin l^{4} \cup e_{3}^{4}$ отсекаются опять-таки теми же рассуждениями, поскольку там получалось противоречие $\bar{\nu}_{5}<n$. Мы, однако, должны дать иные аргументы в ситуации $\bar{B}_{4} \in l^{4}$, так как у нас нет условия $\nu_{5}>\frac{13}{10} n$. Можно предложить следующий подход. Легко видеть, что

$$
2 \alpha_{0}+\alpha_{1}+\cdots+\alpha_{4} \leqslant\left(\left.D\right|_{F}-\mu l\right) \circ l=2 n-2 \mu .
$$

Учитывая, что $\nu_{5}=\mu+\alpha_{5}+m_{4}$, имеем

$$
2 \bar{\nu}_{1}+\bar{\nu}_{2}+\cdots+\bar{\nu}_{5}=8 \mu+2 \alpha_{0}+\alpha_{1}+\cdots+\alpha_{4}+2 m_{1}+m_{2}+m_{3}+m_{4}
$$

откуда $2 \nu_{1}+\nu_{2}+\cdots+\nu_{5} \leqslant 8 n$. Тогда

$$
\nu_{1}+\cdots+\nu_{5} \leqslant \frac{5}{6}\left(2 \nu_{1}+\nu_{2}+\cdots+\nu_{5}\right) \leqslant \frac{20}{3} n
$$

и мы пришли к противоречию: $\frac{69}{50}<\theta_{5} \leqslant \frac{4}{3}$. Случай $L=k=3$ исключен.

Пусть теперь $L=k=2$. Так как $\nu_{3}>\frac{13}{10} n$, то все рассуждения и оценки с нулевого по второй этаж сохраняются. Мы будем использовать предположение, что $\nu_{1} / n \geqslant \theta_{4} \geqslant \theta_{5}>\frac{69}{50}$. 
На третьем этаже либо $\bar{B}_{3} \in e_{1}^{3}$, либо $\bar{B}_{3} \in l^{3}$, либо $\bar{B}_{3} \notin l^{3} \cup e_{1}^{3}$ (ситуация $\bar{B}_{3} \in e_{2}^{3}$ невозможна ввиду п. 4 предложения 1.1).

Итак, пусть $\bar{B}_{3} \in e_{1}^{3}$. Очевидно,

$$
\alpha_{0}+\cdots+\alpha_{3} \leqslant 2 \alpha_{0}+\alpha_{1}+\alpha_{2} \leqslant 2 n-2 \mu \text {. }
$$

Тогда, поскольку $\bar{\nu}_{4}=\alpha_{3}+m_{1}+m_{3}$, имеем

$$
\bar{\nu}_{1}+\cdots+\bar{\nu}_{4}=4 \mu+\alpha_{0}+\cdots+\alpha_{3}+3 m_{1}+m_{2}+m_{3},
$$

откуда $\nu_{1}+\cdots+\nu_{4} \leqslant 4 n+2 m_{1}<\frac{131}{25} n$ (мы использовали, что $m_{1}<\frac{31}{50} n$ ). Таким образом, пришли к противоречию: $\frac{69}{50}<\theta_{4}<\frac{131}{100}$. Ясно, что ситуация $\bar{B}_{3} \notin l^{3} \cup e_{1}^{3}$ тем более невозможна.

Пусть $\bar{B}_{3} \in l^{3}$. Тогда $2 \alpha_{0}+\alpha_{1}+\alpha_{2}+\alpha_{3} \leqslant 2 n-2 \mu$ и

$$
2 \bar{\nu}_{1}+\bar{\nu}_{2}+\bar{\nu}_{3}+\bar{\nu}_{4}=7 \mu+2 \alpha_{0}+\alpha_{1}+\alpha_{2}+\alpha_{3}+2 m_{1}+m_{2}+m_{3} \text {. }
$$

С другой стороны, $2 \nu_{1}+\nu_{2}+\nu_{3}+\nu_{4}>\frac{69}{50} n+4 n \theta_{4}>\frac{69}{10} n$ и мы находим, что

$$
\frac{69}{10} n+m_{4}<7 n
$$

т.е. $m_{4}<\frac{1}{10} n$.

Рассмотрим четвертый этаж. Пусть $\bar{B}_{4} \in e_{3}^{4}$. Тогда

$$
\bar{\nu}_{5}=\alpha_{4}+m_{3}+m_{4}<\frac{3}{25} n+\frac{14}{25} n+\frac{1}{10} n<n,
$$

так как $\alpha_{4} \leqslant \alpha_{2}<\frac{3}{25} n$ и $m_{3}<\frac{14}{25} n$. Очевидно, $\bar{B}_{4} \notin l^{4} \cup e_{3}^{4}$ невозможно по аналогичным соображениям. Пусть $\bar{B}_{4} \in l^{4}$. Тогда

$$
\begin{gathered}
2 \alpha_{0}+\alpha_{1}+\cdots+\alpha_{4} \leqslant 2 n-2 \mu, \\
\bar{\nu}_{5}=\mu+\alpha_{4}+m_{4} .
\end{gathered}
$$

Далее, $2 \bar{\nu}_{1}+\bar{\nu}_{2}+\cdots+\bar{\nu}_{5}=8 \mu+2 \alpha_{0}+\alpha_{1}+\cdots+\alpha_{4}+2 m_{1}+m_{2}+m_{3}+m_{4}$, так что $2 \nu_{1}+\nu_{2}+\cdots+\nu_{5} \leqslant 8 n$, откуда

$$
\nu_{1}+\cdots+\nu_{5} \leqslant \frac{5}{6} \cdot 8 n
$$

т.е. $\theta_{5} \leqslant \frac{4}{3}$, что противоречит условию $\theta_{5}>\frac{69}{50}$. Случай $L=k=2$ исключен.

Таким образом, мы показали, что ситуация $L=k$ не реализуется. Тем самым завершено доказательство теоремы 2.3.

\section{Список литературы}

1. Гриненко M. М. Бирациональные свойства пучков поверхностей дель Пеццо степеней 1 и 2 // Матем. сб. 2000. Т. 191. № 5. С. 17-38. 
2. Пухликов А. В. Бирациональные автоморфизмы трехмерных алгебраических многообразий с пучком поверхностей дель Пеццо // Изв. РАН. Сер. матем. 1998. Т. 62. № 1. C. $123-164$.

3. Соболев И. В. Бирациональные автоморфизмы одного класса многообразий, расслоенных на кубические поверхности // Изв. РАН. Сер. матем. 2002. Т. 66. № 1. С. 203-224.

4. Pukhlikov A. V. Essentials of the method of maximal singularities // Explicit birational geometry of 3-folds. Cambridge: Cambridge Univ. Press, 2000. P. 73-100. (London Math. Soc. Lecture Note Ser. V. 281.)

5. Исковских В. А. Бирациональные автоморфизмы трехмерных алгебраических многообразий // Итоги науки и техники. Совр. проблемы матем. Т. 12. М.: ВИНИТИ, 1979. C. $159-236$.

6. Corti A. Factoring birational maps of threefolds after Sarkisov // J. Algebraic Geom. 1995. V. 4. P. 223-254.

Математический институт

им. В.А. Стеклова РАН

Поступила в редакцию

E-mail: grin@mi.ras.ru

04.06.2002 